\title{
SCATTERING THEORY BELOW ENERGY SPACE FOR TWO DIMENSIONAL NONLINEAR SCHRÖDINGER EQUATION
}

\author{
CHANGXING MIAO AND JIQIANG ZHENG
}

\begin{abstract}
The purpose of this paper is to illustrate the I-method by studying low-regularity solutions of the nonlinear Schrödinger equation in two space dimensions. By applying this method, together with the interaction Morawetz estimate [8] 36, we establish global wellposedness and scattering for low-regularity solutions of the equation $i u_{t}+\Delta u=\lambda_{1}|u|^{p_{1}} u+$ $\lambda_{2}|u|^{p_{2}} u$ under certain assumptions on parameters. This is the first result of this type for an equation which is not scale-invariant. In the first step, we establish global well-posedness and scattering for low regularity solutions of the equation $i u_{t}+\Delta u=|u|^{p} u$, for a suitable range of the exponent $p$ extending the result of Colliander, Grillakis and Tzirakis [Comm. Pure Appl. Math. 62(2009), 920-968.]
\end{abstract}

Key Words: nonlinear Schrödinger equation; global well-posedness; scattering; low regularity.

AMS Classification: 35Q55.

\section{INTRODUCTION}

We consider the initial-value problem for the defocusing nonlinear Schrödinger equation

$$
\left\{\begin{array}{l}
\left(i \partial_{t}+\Delta\right) u=f(u), \quad(t, x) \in \mathbb{R} \times \mathbb{R}^{2} \\
u(0, x)=u_{0}(x)
\end{array}\right.
$$

where $u: \mathbb{R}_{t} \times \mathbb{R}_{x}^{2} \rightarrow \mathbb{C}$. If $f(u)=|u|^{p} u$, the equation in (1.1) is invariant under the scaling transform

$$
u(t, x) \mapsto \lambda^{2 / p} u\left(\lambda^{2} t, \lambda x\right), \quad \text { for any } \lambda>0,
$$

and this scaling property leads to the notion of criticality for problem (1.1). Indeed, one can verify that the homogeneous Sobolev space $\dot{H}^{s_{c}}\left(\mathbb{R}^{2}\right)$ with the critical regularity index $s_{c}:=1-\frac{2}{p}$ is invariant under scaling (1.2). Then, for every $u_{0} \in H_{x}^{s}\left(\mathbb{R}^{2}\right)$, we refer to the problem (1.1) as critical if $s=s_{c}$, subcritical for $s>s_{c}$, and supercritical if $s<s_{c}$.

If a smooth solution $u$ of problem (1.1) has sufficient decay at infinity, it conserves mass

$$
M(u)=\int_{\mathbb{R}^{2}}|u(t, x)|^{2} d x=M\left(u_{0}\right)
$$

and the energy

$$
E(u(t))=\int_{\mathbb{R}^{2}}\left(\frac{1}{2}|\nabla u(t)|^{2}+F(u)\right) d x=E\left(u_{0}\right), F(u)=\int_{0}^{|u|} f(s) d s .
$$

The global well-posedness and scattering theory for the defocusing Schrödinger equation(NLS)

$$
\left\{\begin{array}{l}
\left(i \partial_{t}+\Delta\right) u=|u|^{p} u, \quad(t, x) \in \mathbb{R} \times \mathbb{R}^{d} \\
u(0, x)=u_{0}(x) \in H^{s}\left(\mathbb{R}^{d}\right)
\end{array}\right.
$$


has been intensively studied in papers $1,4,5,7,11,13,14,25,34,35$. . Recall that a global solution $u$ to (1.5) scatters in $H^{s}\left(\mathbb{R}^{d}\right)$, if there exist unique state $u_{ \pm} \in H_{x}^{s}\left(\mathbb{R}^{d}\right)$ such that

$$
\lim _{t \rightarrow \pm \infty}\left\|u(t)-e^{i t \Delta} u_{ \pm}\right\|_{H_{x}^{s}\left(\mathbb{R}^{d}\right)}=0 .
$$

In the energy-subcritical case, i.e. for $p \in\left(\frac{4}{d}, \frac{4}{d-2}\right)$ if $d \geqslant 3$ and for $p \in\left(\frac{4}{d},+\infty\right)$ if $d \in\{1,2\}$, for every $u_{0} \in H^{1}\left(\mathbb{R}^{d}\right)$, it is easy to prove the global well-posedness for problem (1.5) by combining the Strichartz estimate together with a standard fixed point argument and the conservation of energy. Ginibre and Velo [25] proved the scattering in spatial dimension $d \geqslant 3$ by making use of the almost finite propagation speed

$$
\int_{|x| \geqslant a}|u(t, x)|^{2} d x \leqslant \int \min \left(\frac{|x|}{a}, 1\right)\left|u\left(t_{0}\right)\right|^{2} d x+\frac{C}{a} \cdot\left|t-t_{0}\right|
$$

for large spatial scale and the classical Morawetz inequality in 32

$$
\iint_{\mathbb{R} \times \mathbb{R}^{d}} \frac{|u(t, x)|^{p+2}}{|x|} d t d x \lesssim\|u\|_{L_{t}^{\infty} \dot{H}^{\frac{1}{2}}}^{2} \lesssim C\left(M\left(u_{0}\right), E\left(u_{0}\right)\right)
$$

for small spatial scale. It is well known that the Morawetz estimate is an essential tool in the proof of scattering for the nonlinear dispersive equations such as nonlinear Schrödinger equations and nonlinear Klein-Gordon equations. A classical Morawetz inequality was first derived by Morawetz 33] for the nonlinear Klein-Gordon equation, and then extended by Lin and Strauss 32 to the nonlinear Schrödinger equation with $d \geqslant 3$ in order to obtain the scattering for slightly more regular solutions. Next, Nakanishi 35] extended the above Morawetz inequality to the dimensions $d \in\{1,2\}$ by considering certain variants of the Morawetz estimate with space-time weights and consequently he proved the scattering in low dimensions.

The Morawetz estimate (1.6) plays an important role in the proof of scattering for the problem (1.5) in the energy-subcritical case, but it does not work so powerfully in the energycritical case (i.e. for $p=\frac{4}{d-2}$ if $d \geqslant 3$ ). Thus, to obtain the scattering in the critical case, it is a very difficult problem. An essential breakthrough came from Bourgain [3] who exploited the 'induction on energy' technique and the following spatial-localized Morawetz inequality

$$
\int_{I} \int_{|x| \leqslant C|I|^{\frac{1}{2}}} \frac{|u(t, x)|^{6}}{|x|} d t d x \lesssim|I|^{\frac{1}{2}} C\left(\|u\|_{L_{t}^{\infty} \dot{H}^{1}\left(I \times \mathbb{R}^{3}\right)}\right)
$$

to obtain the scattering of radial solutions to problem (1.5) with $p=4$ in the energy space $\dot{H}^{1}\left(\mathbb{R}^{3}\right)$. Next, Colliander, Keel, Staffilani, Takaoka and Tao (I-team) 17 removed the radial symmetry assumption in [3], and solved this longtime standing problem through the Bourgain 'induction on energy' technique and the frequency localized type of the interaction Morawetz inequality

$$
\left\||\nabla|^{\frac{3-d}{2}}\left(|u|^{2}\right)\right\|_{L_{t, x}^{2}\left(\mathbb{R} \times \mathbb{R}^{d}\right)}^{2} \lesssim\left\|u_{0}\right\|_{L_{x}^{2}}^{2}\|u\|_{L_{t}^{\infty}\left(\mathbb{R} ; \dot{H}^{\frac{1}{2}}\right)}^{2}, \quad d \geqslant 1 .
$$

This interaction Morawetz inequality was first derived by I-team in their work [16] in spatial dimension $d=3$ and then extended to $d \geqslant 4$ in [39]. Colliander, Grillakis and Tzirakis [8], Planchon and Vega 36 independently proved (1.8) in dimensions $d \in\{1,2\}$. As a byproduct, one can easily give another simpler proof of the result of Ginibre and Velo [25, see [39] for more detail. We also refer the reader to [27] for the exposition on the Morawetz inequalities and their applications.

The interaction Morawetz inequality plays also an important role in the study of a low regularity problem. Where we ask what is the minimal $s$ to ensure that problem (1.5) has either a local solution or a global solution for which the scattering hold? Such a problem was first considered by Cazenave and Weissler [5], who proved that problem (1.5) is locally well posed in $H^{s}\left(\mathbb{R}^{d}\right)$ with $s \geqslant \max \left\{0, s_{c}\right\}$ and globally well posed together with scattering for small 
data in $\dot{H}^{s_{c}}\left(\mathbb{R}^{d}\right)$ with $s_{c} \geqslant 0$. They used Strichartz estimates in the framework of Besov spaces. On the other hand, since the lifespan of local solutions depend only on the $H^{s}$-norm of the initial data for $s>\max \left\{0, s_{c}\right\}$, one can easily obtain the global well-posedness for (1.5) in two special cases: the mass subcritical case $\left(p<\frac{4}{d}\right)$ for $L_{x}^{2}\left(\mathbb{R}^{d}\right)$-initial data and the energysubcritical case (for $p<\frac{4}{d-2}$, if $d \geqslant 3$ or for $p<+\infty$ if $d \in\{1,2\}$ ) for $H_{x}^{1}\left(\mathbb{R}^{d}\right.$ )-initial data by using the conservation of mass and energy respectively.

This leaves the open problem on global well-posedness in $H^{s}\left(\mathbb{R}^{d}\right)$ in the intermediate regime $0 \leqslant s_{c} \leqslant s<1$. The first progress on this direction came from the Bourgain 'Fourier truncation method [1] where refinements of Strichartz' inequality [2], high-low frequency decompositions and perturbation methods were used to show that problem (1.5) with $p=2$ is globally wellposed in $H^{s}\left(\mathbb{R}^{3}\right)$ with $s>\frac{11}{13}$ such that

$$
u(t)-e^{i t \Delta} u_{0} \in H^{1}\left(\mathbb{R}^{3}\right)
$$

This leads to the I-method which was derived by Keel and Tao in the study of wave maps [29]. Subsequently, I-team developed the I-method to treat many low regularity problems including the nonlinear Schrödinger equations with derivatives, the one dimensional quintic NLS, and the cubic NLS in two and three dimensions [10 15. Compared with the result in [1, I-team also obtained the scattering in $H^{s}\left(\mathbb{R}^{3}\right)$ with $s>\frac{5}{6}$ by using the I-method and the interaction Morawetz estimate (1.8) in [16]. Dodson [20] extended those results to $s>\frac{5}{7}$ by means of a linear-nonlinear decomposition, and then $\mathrm{Su} 38$ to $s>\frac{2}{3}$. For the cubic NLS in dimension two (corresponding to the mass-critical), I-term further exploited the improved I-method in [15] to get the global well-posedness for $s>\frac{1}{2}$. Colliander, Grillakis and Tzirakis [7] extended it to $s>\frac{2}{5}$ by means of the I-method and the improved interaction Morawetz inequalities. Laterly, Colliander and Roy [18] improved these results to $s>\frac{1}{3}$. Subsequently, Dodson [19] showed the global well-posedness for $s>\frac{1}{4}$ by improving the almost Morawetz estimates from [7.

The study of a low regularity problem stimulates the development of the scattering in $L^{2}\left(\mathbb{R}^{d}\right)$ for the mass-critical problem (i.e. for $p=\frac{4}{d}$ ). Dodson 22 24 developed so called long-time Strichartz estimates to prove the global well-posedness and scattering in $L_{x}^{2}$-space by making use of a concentration-compactness approach and the idea of I-method.

Now, let us describe the I-method, which consists in smoothing out the $H^{s}$-initial data with $0<s<1$ in order to access a good local and global theory available at the $H^{1}$-regularity. To do it, we define the Fourier multiplier $I$ by

$$
\widehat{I u}(\xi):=m(\xi) \hat{u}(\xi),
$$

where $m(\xi)$ is a smooth radial decreasing cut off function such that

$$
m(\xi)= \begin{cases}1, & |\xi| \leqslant N, \\ \left(\frac{|\xi|}{N}\right)^{s-1}, & |\xi| \geqslant 2 N .\end{cases}
$$

Thus, $I$ is the identity operator on frequencies $|\xi| \leqslant N$ and behaves like a fractional integral operator of order $1-s$ on higher frequencies. It is easy to show that the operator $I$ maps $H^{s}$ to $H^{1}$. Moreover, we have

$$
\|u\|_{H^{s}} \lesssim\|I u\|_{H^{1}} \lesssim N^{1-s}\|u\|_{H^{s}} .
$$

Thus, to prove that problem (1.5) is globally well-posed in $H^{s}\left(\mathbb{R}^{d}\right)$, it suffices to show that $E(I u(t))<+\infty$ for all $t \in \mathbb{R}$. Since $I u$ is not a solution to (1.5), the modified energy $E(I u)(t)$ is not conserved. Indeed, we have

$$
\frac{d}{d t} E(I u(t))=\operatorname{Re} \int_{\mathbb{R}^{d}} \overline{I u_{t}}\left[|I u|^{p} I u-I\left(|u|^{p} u\right)\right] d x
$$


Thus, the key idea is to show that the modified energy $E(I u)$ is an 'almost conserved' quantity in the sense that its derivative $\frac{d}{d t} E(I u(t))$ will decay with respect to a large parameter $N$. This will allow us to control $E(I u)$ on time interval where the local solution exists and we can iterate this estimate to obtain a global in time control of the solution by means of the bootstrap argument, see Section 3 for more details. Then immediately we get a bound for the $H^{1}$-norm of $I u$ which will give us an $H^{s}$-bound for the solution $u$ by inequality (1.11).

To deal with equality (1.12), one needs complicated estimates on the commutator $I\left(|u|^{p} u\right)-$ $|I u|^{p} I u$. When $p$ is an even integer, one can write the commutator explicitly by means of the Fourier transform and to control it by multilinear harmonic analysis, see [7, 9, 15, 18, 21, 38, 40 for considerations of the algebraic nonlinearity $f(u)=|u|^{2 k} u$ with $k \in \mathbb{N}$ in $\mathbb{R}^{d}(d=1,2)$ and cubic NLS in $\mathbb{R}^{3}$. Colliander, Grillakis and Tzirakis 8 proved that a solution to (1.5) with $f(u)=|u|^{2 k} u$ is global and scatters for $s>1-\frac{1}{4 k-3}$ in $\mathbb{R}^{2}$. Recently, by exploiting the long-time Strichartz estimate in the Koch-Tataru space $U_{\Delta}^{2}$ and $V_{\Delta}^{2}$ (see [30,31]), Dodson [21] extended this result to $s>1-\frac{1}{k}$ for radial initial data.

Unfortunately, the above method for estimating (1.12) depends heavily on the exact form of the nonlinearity. Therefore, this method fails when $p$ is not an even integer. For arbitrary $p \in(0,4 /(d-2))$ and $d \geqslant 3$, by relying on more rudimentary tools as Taylor's expansion and Strichartz estimates, I-team 14 obtained polynomial growth of the $H^{s}$-norm of solutions, and so the global well-posedness for problem (1.5) with $s$ sufficiently close to 1 . However, their bounds are insufficient to yield scattering. Subsequently, Visan and Zhang [41] combined the I-method and the a priori interaction Morawetz estimate (1.8) to show that scattering holds in $H^{s}\left(\mathbb{R}^{d}\right)(d \geqslant 3)$ for $s$ being larger than some $s_{0}(d, p) \in(0,1)$. This method is weaker than the multilinear multiplier method when $p$ is an even integer.

I-method also relies on the scale-invariance of the equation in (1.5). Therefore, adding a perturbation to the equation which destroys the scale invariance, is of particular interest. By this reason, we study the nonlinear Schrödinger equation (1.5) which is perturbed by a lowerorder nonlinearity

$$
\left\{\begin{array}{l}
\left(i \partial_{t}+\Delta\right) u=|u|^{p_{1}} u+|u|^{p_{2}} u, \quad(t, x) \in \mathbb{R} \times \mathbb{R}^{2}, p_{1}<p_{2}, \\
u(0, x)=u_{0}(x) .
\end{array}\right.
$$

We look for answers to the following questions: under which conditions on $p_{1}$ and $p_{2}$ a solution to problem (1.13) is unique global in time in $H^{s}\left(\mathbb{R}^{2}\right)$ with suitable $s$, and is scattering? We use a certain perturbative and scale technique. We first remove the term $|u|^{p_{2}} u$ and study the global well-posedness and scattering for (1.1) with general nonlinearity $f(u)=|u|^{p} u$ by arguments of 41] combined with the a priori interaction Morawetz estimates in [8, 36]. Then, we apply the I-method to an equation derived from that in (1.13) by the scaling transform (1.2).

Now, we collect our results into the following theorems. We define

$$
s_{0}:=\max \left\{\frac{1+s_{c}}{2}, \frac{p}{p+1}, \quad s_{1}\right\}, s_{c}=1-\frac{2}{p},
$$

and $s_{1}$ is the positive root of the quadratic equation

$$
s^{2}+2 s_{c} s+s_{c}^{2}-4 s_{c}=0 .
$$

Theorem 1.1. Assume that $u_{0} \in H^{s}\left(\mathbb{R}^{2}\right)$ with $s \in\left(s_{0}, 1\right), p \geqslant \frac{11}{4}$. Then the solution $u$ to (1.1) with $f(u)=|u|^{p} u$ is global and scatters in the sense that there exist unique $u_{ \pm} \in H_{x}^{s}\left(\mathbb{R}^{2}\right)$ such that

$$
\lim _{t \rightarrow \pm \infty}\left\|u(t)-e^{i t \Delta} u_{ \pm}\right\|_{H_{x}^{s}\left(\mathbb{R}^{2}\right)}=0
$$

Remark 1.1. There exists a gap for the region $2<p<\frac{11}{4}$. The restriction to $p \geqslant \frac{11}{4}$ comes from estimate (3.9) in Proposition 3.1 and estimate (3.24) in Proposition 3.2, since the classical 
interaction Morawetz estimates are not good enough to control this time-space norm. We refer to Propositions 3.1 and 3.2 for more detail.

Now, we want to deal with the case $f(u)=|u|^{p} u$ with $p<\frac{11}{4}$. We will apply the following improved interaction Morawetz estimates in [7]

$$
\int_{0}^{T} \int_{\mathbb{R}^{2}}|u(t, x)|^{4} d x d t \lesssim T^{\frac{1}{3}}\left\|u_{0}\right\|_{L_{x}^{2}}^{2}\|u\|_{L_{t}^{\infty}\left([0, T], \dot{H}_{x}^{1 / 2}\right)}^{2}+T^{\frac{1}{3}}\left\|u_{0}\right\|_{L_{x}^{2}}^{4}
$$

instead of the following classical interaction Morawetz estimates in [8, 36]

$$
\|u\|_{L_{t, x}^{5}\left(I \times \mathbb{R}^{2}\right)} \lesssim\|u\|_{L_{t}^{\infty}\left(I ; \dot{H}^{\frac{1}{2}}\left(\mathbb{R}^{2}\right)\right)}^{\frac{2}{5}}\left\|u_{0}\right\|_{2}^{\frac{3}{5}}
$$

The estimate (1.16) will help us to obtain global well-posedness with the lower order $p$. But the $H^{s}$-norm of the solution depends on the polynomial growth of time, which is insufficient to yield scattering. Let us define

$$
\tilde{s}_{0}:=\max \left\{\frac{1+s_{c}}{2}, \frac{p}{p+1}, \tilde{s}_{1}\right\}, s_{c}=1-\frac{2}{p},
$$

and $\tilde{s}_{1}$ be the positive root of the quadratic equation

$$
3\left(s-s_{c}\right)^{2}-2\left(1+6 s_{c}\right)(1-s)=0 .
$$

Theorem 1.2. Assume that $u_{0} \in H^{s}\left(\mathbb{R}^{2}\right)$ with $s \in\left(\tilde{s}_{0}, 1\right)$ and $p>2$. Then the solution $u$ to (1.1) with $f(u)=|u|^{p} u$ is global. Furthermore, we have the polynomial growth of the $H^{s}$-norm of the solution,

$$
\sup _{t \in[0, T]}\|u(t)\|_{H^{s}\left(\mathbb{R}^{2}\right)} \leqslant C\left(\left\|u_{0}\right\|_{H^{s}\left(\mathbb{R}^{2}\right)}\right)(1+T)^{\frac{1-s}{3\left(s-s_{c}\right)^{2}-2\left(1+6 s_{c}\right)(1-s)}}+, \quad \forall T>0 .
$$

Now we turn to problem (1.13) with $p_{2}=2 k, k \in \mathbb{N}$ and $p_{1}=p$. Denote

$$
s_{c}^{(1)}=1-\frac{2}{p}, s_{c}^{(2)}=1-\frac{1}{k},
$$

and

$$
\tilde{s}_{3}:=\max \left\{\frac{1+s_{c}^{(1)}}{2}, \frac{2 k}{2 k+1}, \frac{5 s_{c}^{(2)}}{4 s_{c}^{(2)}+1}, s_{3}\right\}
$$

where $s_{3}$ is the positive root of the quadratic equation

$$
s^{2}-\left(s_{c}^{(1)}+s_{c}^{(2)}-\alpha\right) s-\alpha=0, \alpha=4 s_{c}^{(2)}-\frac{9\left(2-\frac{p}{k}\right)}{2(p+2)} .
$$

Theorem 1.3. Assume that $u_{0} \in H^{s}\left(\mathbb{R}^{2}\right)$ with

$$
s \in\left(\tilde{s}_{3}, 1\right), 2 k>p \geqslant \frac{11}{4} \text { and } 1<k \in \mathbb{N} .
$$

Then the solution $u$ to (1.1) with $f(u)=|u|^{2 k} u+|u|^{p} u$ is global and scatters in $H^{s}\left(\mathbb{R}^{2}\right)$.

Remark 1.2. A simple computation shows $\tilde{s}_{3}>s_{0}$ here. Our argument also works in the higher dimensional case. By the same way as in the proof of Theorem 1.2, one can also use the improved interaction Morawetz estimates (1.16) to achieve the global well-posedness of (1.13) with $p \in\left[2, \frac{11}{4}\right]$.

Finally, we give the global well-posedness and scattering result for (1.1) with more general nonlinearity $f(u)=|u|^{p_{1}} u+|u|^{p_{2}} u$ by the same arguments as those in the proofs of Theorems 1.1 and 1.3 
Theorem 1.4. Assume that $u_{0} \in H^{s}\left(\mathbb{R}^{2}\right)$ with

$$
s \in\left(\max \left\{\frac{1+s_{c}^{(2)}}{2}, \frac{p_{2}}{p_{2}+1}, s_{2}\right\}, 1\right), s_{c}^{(j)}=1-\frac{2}{p_{j}}, j=1,2, \frac{11}{4} \leqslant p_{1}<p_{2}
$$

and $s_{2}$ is the positive root of the quadratic equation

$$
s^{2}+2 s_{c}^{(2)} s+\left(s_{c}^{(2)}\right)^{2}-4 s_{c}^{(2)}=0 .
$$

Then the solution of problem (1.1) with $f(u)=|u|^{p_{1}} u+|u|^{p_{2}} u$ is global and scatters in $H^{s}\left(\mathbb{R}^{2}\right)$.

The paper is organized as follows. In Section 2, as preliminaries, we gather some notations and recall the Strichartz estimate for NLS and some nonlinear estimates. In Section 3, we will prove Theorem 1.1 by making use of I-method together with the interaction Morawetz estimates. In Section 4, we will utilize I-method and the improved interaction Morawetz inequalities to show Theorem 1.2. We prove Theorem 1.3 in Section 5 based on Theorem 1.1. In Appendix, we state a result in one dimension.

\section{Preliminaries}

2.1. Notations. To simplify our inequalities, we introduce the symbols $\lesssim, \sim, \ll$. If $X, Y$ are nonnegative quantities, we write either $X \lesssim Y$ or $X=\mathcal{O}(Y)$ to denote the estimate $X \leqslant C Y$ for some $C$, and $X \sim Y$ to denote the estimate $X \lesssim Y \lesssim X$. We use $X \ll Y$ to mean $X \leqslant c Y$ for some small constant $c$. We use $C \gg 1$ to denote various large finite constants, and $0<c \ll 1$ to denote various small constants. For every $r$ such that $1 \leqslant r \leqslant \infty$, we denote by $\|\cdot\|_{r}$ the norm in the Lebesgue space $L^{r}=L^{r}\left(\mathbb{R}^{d}\right)$ and by $r^{\prime}$ the conjugate exponent defined by $\frac{1}{r}+\frac{1}{r^{\prime}}=1$. We denote by $a \pm$ quantities of the form $a \pm \epsilon$ for any $\epsilon>0$. We always assume $d=2$ and $s<1$.

Let $f(z):=|z|^{p} z$, then

$$
f_{z}(z):=\frac{\partial f}{\partial z}(z)=\frac{p+2}{2}|z|^{p} \text { and } f_{\bar{z}}(z):=\frac{\partial f}{\partial \bar{z}}(z)=\frac{p}{2}|z|^{p} \frac{z}{\bar{z}} .
$$

We denote $F^{\prime}$ to be the vector $\left(f_{z}, f_{\bar{z}}\right)$ and use the notation

$$
w \cdot f^{\prime}(z)=w f_{z}(z)+\bar{w} f_{\bar{z}}(z) .
$$

In particular, we get by the chain rule

$$
\nabla f(u)=\nabla u \cdot f^{\prime}(u),
$$

and

$$
\left|f^{\prime}(z)-f^{\prime}(w)\right| \lesssim|z-w|(|z|+|w|)^{p-1}, \quad p>1
$$

The Fourier transform on $\mathbb{R}^{2}$ is defined by

$$
\widehat{f}(\xi):=(2 \pi)^{-1} \int_{\mathbb{R}^{2}} e^{-i x \cdot \xi} f(x) d x,
$$

giving rise to the fractional differentiation operators $|\nabla|^{s}$ and $\langle\nabla\rangle^{s}$ defined by

$$
\widehat{|\nabla|^{s} f}(\xi):=|\xi|^{s} \hat{f}(\xi), \widehat{\langle\nabla\rangle^{s} f}(\xi):=\langle\xi\rangle^{s} \hat{f}(\xi),
$$

where $\langle\xi\rangle:=1+|\xi|$. This helps us to define the homogeneous and inhomogeneous Sobolev norms

$$
\|f\|_{\dot{H}_{x}^{s}\left(\mathbb{R}^{2}\right)}:=\left\||\xi|^{s} \hat{f}\right\|_{L_{x}^{2}\left(\mathbb{R}^{2}\right)},\|f\|_{H_{x}^{s}\left(\mathbb{R}^{2}\right)}:=\left\|\langle\xi\rangle^{s} \hat{f}\right\|_{L_{x}^{2}\left(\mathbb{R}^{2}\right)} .
$$

We will also need the Littlewood-Paley projection operators. Let $\varphi(\xi)$ be a smooth bump function adapted to the ball $|\xi| \leqslant 2$ which equals 1 on the ball $|\xi| \leqslant 1$. For each dyadic number 
$N \in 2^{\mathbb{Z}}$, we define the Littlewood-Paley operators

$$
\begin{aligned}
\widehat{P_{\leqslant N} f}(\xi) & :=\varphi\left(\frac{\xi}{N}\right) \widehat{f}(\xi), \\
\widehat{P_{>N} f}(\xi) & :=\left(1-\varphi\left(\frac{\xi}{N}\right)\right) \widehat{f}(\xi), \\
\widehat{P_{N} f}(\xi) & :=\left(\varphi\left(\frac{\xi}{N}\right)-\varphi\left(\frac{2 \xi}{N}\right)\right) \widehat{f}(\xi) .
\end{aligned}
$$

Similarly, we can define $P_{<N}, P_{\geqslant N}$, and $P_{M<\leqslant N}=P_{\leqslant N}-P_{\leqslant M}$, whenever $M$ and $N$ are dyadic numbers. Especially, we denote $P_{1}:=P_{\leqslant 1}$. We will frequently write $f_{\leqslant N}$ for $P_{\leqslant N} f$ and similarly for the other operators.

The Littlewood-Paley operators commute with derivative operators, the free propagator, and the conjugation operation. They are self-adjoint and bounded on every space $L_{x}^{p}\left(\mathbb{R}^{2}\right)$ and $\dot{H}_{x}^{s}\left(\mathbb{R}^{2}\right)$ for $1 \leqslant p \leqslant \infty$ and $s \geqslant 0$. Moreover, they also obey the following Bernstein estimates.

Lemma 2.1 (Bernstein estimates). For every $s \geqslant 0,1 \leqslant p \leqslant q \leqslant \infty$, and $N \in \mathbb{N}$, we have

$$
\begin{aligned}
& \left\|P_{\geqslant N} f\right\|_{L^{p}\left(\mathbb{R}^{2}\right)} \lesssim N^{-s}\left\||\nabla|^{s} P_{\geqslant N} f\right\|_{L^{p}\left(\mathbb{R}^{2}\right)}, \\
& \left\||\nabla|^{s} P_{\leqslant N} f\right\|_{L^{p}\left(\mathbb{R}^{2}\right)} \lesssim N^{s}\left\|P_{\leqslant N} f\right\|_{L^{p}\left(\mathbb{R}^{2}\right)}, \\
& \left\||\nabla|^{ \pm s} P_{N} f\right\|_{L^{p}\left(\mathbb{R}^{2}\right)} \sim N^{ \pm s}\left\|P_{N} f\right\|_{L^{p}\left(\mathbb{R}^{2}\right)}, \\
& \left\|P_{\leqslant N} f\right\|_{L^{q}\left(\mathbb{R}^{2}\right)} \lesssim N^{\frac{2}{p}-\frac{2}{q}}\left\|P_{\leqslant N} f\right\|_{L^{p}\left(\mathbb{R}^{2}\right)}, \\
& \left\|P_{N} f\right\|_{L^{q}\left(\mathbb{R}^{2}\right)} \lesssim N^{\frac{2}{p}-\frac{2}{q}}\left\|P_{N} f\right\|_{L^{p}\left(\mathbb{R}^{2}\right)} .
\end{aligned}
$$

2.2. Strichartz estimates. Let $e^{i t \Delta}$ be the free Schrödinger propagator given by

$$
\left[e^{i t \Delta} f\right](x)=\frac{1}{4 \pi i t} \int_{\mathbb{R}^{2}} e^{i|x-y|^{2} / 4 t} f(y) d y, \quad t \neq 0 .
$$

Obviously, it satisfies the dispersive estimate

$$
\left\|e^{i t \Delta} f\right\|_{L_{x}^{\infty}\left(\mathbb{R}^{2}\right)} \lesssim|t|^{-1}\|f\|_{L_{x}^{1}\left(\mathbb{R}^{2}\right)}, \quad t \neq 0 .
$$

Interpolating above inequality with $\left\|e^{i t \Delta} f\right\|_{L_{x}^{2}\left(\mathbb{R}^{2}\right)} \equiv\|f\|_{L_{x}^{2}\left(\mathbb{R}^{2}\right)}$ then yields

$$
\left\|e^{i t \Delta} f\right\|_{L_{x}^{q}\left(\mathbb{R}^{2}\right)} \leqslant C|t|^{-\left(1-\frac{2}{q}\right)}\|f\|_{L_{x}^{q^{\prime}}\left(\mathbb{R}^{2}\right)}, \quad t \neq 0
$$

for $2 \leqslant q \leqslant \infty$. This inequality implies the classical Strichartz estimates by the standard $T T^{*}$ argument, which we will state below. First, we need the following definition.

Definition 2.1 (Admissible pairs). A pair of exponents $(q, r)$ is called Schrödinger admissible in $\mathbb{R}^{2}$, which we denote by $(q, r) \in \Lambda_{0}$ if

$$
2 \leqslant q, r \leqslant \infty, \frac{1}{q}+\frac{1}{r}=\frac{1}{2} \text {, and }(q, r) \neq(2, \infty) .
$$

For a spacetime slab $I \times \mathbb{R}^{2}$, we define the Strichartz norm

$$
\|u\|_{S^{0}(I)}:=\sup \left\{\|u\|_{L_{t}^{q} L_{x}^{r}\left(I \times \mathbb{R}^{2}\right)}:(q, r) \in \Lambda_{0}, q \geqslant 2+\epsilon_{0}\right\},
$$

where $0<\epsilon \ll 1_{0}$. We denote $S^{0}(I)$ to be the closure of all test functions under this norm.

We now state the standard Strichartz estimates in the form that we will need later.

Proposition 2.1 (Strichartz estimates [26, 28, 37]). Let $s \geqslant 0$ and suppose $u: I \times \mathbb{R}^{2} \rightarrow \mathbf{C}$ is a solution to $\left(i \partial_{t}+\Delta\right) u=\sum_{j=1}^{m} F_{j}$. Then

$$
\left\||\nabla|^{s} u\right\|_{S^{0}(I)} \lesssim\left\||\nabla|^{s} u\left(t_{0}\right)\right\|_{L_{x}^{2}\left(\mathbb{R}^{2}\right)}+\sum_{j=1}^{m}\left\|\left.\nabla\right|^{s} F_{j}\right\|_{L_{t}^{q_{j}^{\prime}} L_{x}^{r_{j}^{\prime}}\left(I \times \mathbb{R}^{2}\right)}
$$


for any admissible pairs $\left(q_{j}, r_{j}\right)$ and $t_{0} \in I$.

2.3. Nonlinear estimate. For $N>1$, we define the Fourier multiplier $I:=I_{N}$ given by

$$
\widehat{\operatorname{Iu}}(\xi):=m(\xi) \hat{u}(\xi),
$$

where $m(\xi)$ is a smooth radial decreasing cut off function by (1.10). Let us collect basic properties of $I$.

Lemma 2.2 ( [41]). Let $1<p<\infty$ and $0 \leqslant \sigma \leqslant s<1$. Then,

$$
\begin{aligned}
\|I f\|_{L^{p}} & \lesssim\|f\|_{L^{p}}, \\
\left\||\nabla|^{\sigma} P_{>N} f\right\|_{L^{p}} & \lesssim N^{\sigma-1}\|\nabla I f\|_{L^{p}}, \\
\|f\|_{H^{s}} \lesssim\|I f\|_{H^{1}} & \lesssim N^{1-s}\|f\|_{H^{s}} .
\end{aligned}
$$

We will also need the following fractional calculus estimates.

Lemma 2.3 ( 6]). (i) (Fractional product rule) Let $s \geqslant 0$, and $1<r, r_{j}, q_{j}<\infty$ satisfy $\frac{1}{r}=\frac{1}{r_{i}}+\frac{1}{q_{i}}$ for $i=1,2$. Then

$$
\left\||\nabla|^{s}(f g)\right\|_{L_{x}^{r}\left(\mathbb{R}^{2}\right)} \lesssim\|f\|_{L_{x}^{r_{1}\left(\mathbb{R}^{2}\right)}}\left\||\nabla|^{s} g\right\|_{L_{x}^{q_{1}\left(\mathbb{R}^{2}\right)}}+\left\||\nabla|^{s} f\right\|_{L_{x}^{r_{2}\left(\mathbb{R}^{2}\right)}}\|g\|_{L_{x}^{q_{2}\left(\mathbb{R}^{2}\right)}}
$$

(ii) (Fractional chain rule) Let $G \in C^{1}(\mathbb{C}), s \in(0,1]$, and $1<r, r_{1}, r_{2}<+\infty$ satisfy $\frac{1}{r}=\frac{1}{r_{1}}+\frac{1}{r_{2}}$. Then

$$
\left\||\nabla|{ }^{s} G(u)\right\|_{r} \lesssim\left\|G^{\prime}(u)\right\|_{r_{1}}\left\||\nabla|^{s} u\right\|_{r_{2}}
$$

As noted in the introduction, one needs to estimate the commutator $|I u|^{p} I u-I\left(|u|^{p} u\right)$ in the increment of modified energy $E(I u)(t)$. When $p$ is an even integer, one can use multilinear analysis to expand this commutator into a product of Fourier transforms of $u$ and $I u$, and carefully measure frequency interactions to derive an estimate (see for example [8]). However, this is not possible when $p$ in not an even integer. Instead, Visan and Zhang in 41 established the following rougher estimate:

Lemma 2.4 ( [41]). Let $1<r, r_{1}, r_{2}<\infty$ be such that $\frac{1}{r}=\frac{1}{r_{1}}+\frac{1}{r_{2}}$ and let $0<\nu<s$. Then,

$$
\|I(f g)-(I f) g\|_{L^{r}} \lesssim N^{-(1-s+\nu)}\|I f\|_{L^{r_{1}}}\left\|\langle\nabla\rangle^{1-s+\nu} g\right\|_{L^{r_{2}}}
$$

Furthermore, we have

$$
\left\|\nabla I f(u)-(I \nabla u) f^{\prime}(u)\right\|_{L^{r}} \lesssim N^{-(1-s+\nu)}\|\nabla I u\|_{L^{r_{1}}}\left\|\langle\nabla\rangle^{1-s+\nu} f^{\prime}(u)\right\|_{L^{r_{2}}},
$$

and

$$
\|\nabla I f(u)\|_{L^{r}} \lesssim\|\nabla I u\|_{L^{r_{1}}}\left\|f^{\prime}(u)\right\|_{L^{r_{2}}}+N^{-1+s-\nu}\|\nabla I u\|_{L^{r_{1}}}\left\|\langle\nabla\rangle^{1-s+\nu} f^{\prime}(u)\right\|_{L^{r_{2}}} .
$$

Finally, we conclude this section by recalling the interaction Morawetz estimate for a solution to problem (1.1).

Lemma 2.5 (Interaction Morawetz estimates [8] 36]). Let $u$ be an $H^{\frac{1}{2}}$-solution to (1.1) on the spacetime slab $I \times \mathbb{R}^{2}$. Then, for any $t_{0} \in I$, we have

$$
\|u\|_{L_{t}^{4} L_{x}^{8}\left(I \times \mathbb{R}^{2}\right)}^{4} \lesssim\|u\|_{L_{t}^{\infty}\left(I ; \dot{H}^{\frac{1}{2}}\left(\mathbb{R}^{2}\right)\right)}^{2}\left\|u\left(t_{0}\right)\right\|_{2}^{2}
$$

Moreover, interpolating with $\|u\|_{L_{t}^{\infty} L_{x}^{2}}$, we obtain

$$
\|u\|_{L_{t, x}^{5}\left(I \times \mathbb{R}^{2}\right)}^{5} \lesssim\|u\|_{L_{t}^{\infty}\left(I ; \dot{H}^{\frac{1}{2}}\left(\mathbb{R}^{2}\right)\right)}^{2}\left\|u\left(t_{0}\right)\right\|_{2}^{3} .
$$

Remark 2.1. We adopt the $L_{t, x}^{5}$ interaction Morawetz norm, but not the $L_{t}^{4} L_{x}^{8}$-norm as used in [8]. As we will see in the next section, one needs more restriction on $p$ to use the $L_{t}^{4} L_{x}^{8}$ norm instead of the $L_{t, x}^{5}$ norm. We refer reader to Remark 3.1 for more details. 
To treat the case of low power $p$, we also need the following improved interaction Morawetz inequalities.

Lemma 2.6 (Improved interaction Morawetz estimates [7). Let $u$ be an $H^{\frac{1}{2}}$-solution to (1.1) on the spacetime slab $I \times \mathbb{R}^{2}$. Then, for any $t_{0} \in I$

$$
\|u(t, x)\|_{L_{t, x}^{4}\left(I \times \mathbb{R}^{2}\right)}^{4} \lesssim T^{\frac{1}{3}}\|u\|_{L_{t}^{\infty}\left(I ; \dot{H}^{\frac{1}{2}}\left(\mathbb{R}^{2}\right)\right)}^{2}\left\|u\left(t_{0}\right)\right\|_{2}^{2}+T^{\frac{1}{3}}\left\|u\left(t_{0}\right)\right\|_{2}^{4}
$$

\section{Proof of Theorem 1.1}

In this section, we will use the I-method and the interaction Morawetz estimate to prove Theorem 1.1. At the first step, we need to show that the modified energy $E(I u)$

$$
E(I u)(t)=\frac{1}{2} \int_{\mathbb{R}^{2}}|\nabla I u(t)|^{2}+\frac{1}{p+2} \int_{\mathbb{R}^{2}}|I u(t)|^{p+2}
$$

is an "almost conserved" quantity in the sense that its derivative decays with respect to $N$. In the following, we always assume $s<1$.

3.1. Almost Conservation Law. The aim of this subsection is to control the growth in time of $E(I u)(t)$. First, We define $Z_{I}(t)$ by

$$
Z_{I}(t):=\|I u\|_{Z(t)}=\sup _{(q, r) \in \Lambda_{0}}\left(\sum_{N \geqslant 1}\left\|\nabla P_{N} I u(t)\right\|_{L_{t}^{q} L_{x}^{r}\left(\left[t_{0}, t\right) \times \mathbb{R}^{2}\right)}^{2}\right)^{\frac{1}{2}}
$$

with convention that $P_{1}=P_{\leqslant 1}$. We have the following control of $Z_{I}(t)$.

Proposition 3.1 (The control of $\left.Z_{I}(t)\right)$. Let $u(t, x)$ be an $H^{s}$ solution to problem (1.1) with $f(u)=|u|^{p} u$ defined on $\left[t_{0}, T\right] \times \mathbb{R}^{2}$ and such that

$$
\|u\|_{L_{t, x}^{5}\left(\left[t_{0}, T\right] \times \mathbb{R}^{2}\right)} \leqslant \eta
$$

for some small constant $\eta$. Assume $E\left(I u\left(t_{0}\right)\right) \leqslant 1$. Then for $s>\frac{1+s_{c}}{2}, p \geqslant \frac{5}{2}$, and sufficiently large $N$, we have for any $t \in\left[t_{0}, T\right]$

$$
Z_{I}(t) \lesssim\left\|\nabla I u\left(t_{0}\right)\right\|_{2}+g(t)^{p} Z_{I}(t)+N^{-\left(s-s_{c}\right)} Z_{I}(t) g(t)^{p-1}[g(t)+h(t)],
$$

where $g(t)$ and $h(t)$ are defined as follows:

$$
g(t)^{p}=\eta^{\theta_{1} p} \sup _{s \in\left[t_{0}, t\right]} E(I u(s))^{\frac{\left(1-\theta_{1}\right) p}{p+2}}+\eta^{\theta_{2} p} Z_{I}(t)^{\left(1-\theta_{2}\right) p}+N^{-\left(1-s_{c}\right) p} Z_{I}(t)^{p}
$$

and

$$
h(t)=\eta^{\theta_{1}} \sup _{s \in\left[t_{0}, t\right]} E(I u(s))^{\frac{1-\theta_{1}}{p+2}}+Z_{I}(t)
$$

with $\theta_{1}=\frac{5}{2 p}, \theta_{2}=\frac{5}{2(3 p-5)} \in(0,1)$.

Proof. Applying the operator $I$ to (1.1) and using the Strichartz estimate, we obtain for all $t \in\left[t_{0}, T\right]$

$$
\begin{aligned}
Z_{I}(t) \lesssim & \left\|\nabla I u\left(t_{0}\right)\right\|_{2}+\|\nabla I f(u)\|_{L_{t, x}^{\frac{4}{3}}\left(\left[t_{0}, t\right) \times \mathbb{R}^{2}\right)} \\
\lesssim & \left\|\nabla I u\left(t_{0}\right)\right\|_{2}+\left\|(\nabla I u) f^{\prime}(u)\right\|_{L_{t, x}^{\frac{4}{3}}\left(\left[t_{0}, t\right) \times \mathbb{R}^{2}\right)} \\
& +\left\|\nabla I F(u)-(\nabla I u) f^{\prime}(u)\right\|_{L_{t, x}^{\frac{4}{3}}\left(\left[t_{0}, t\right) \times \mathbb{R}^{2}\right)} \\
\triangleq & \left\|\nabla I u\left(t_{0}\right)\right\|_{2}+I I_{1}+I I_{2} .
\end{aligned}
$$

Throughout the following proof all spacetime norms will be computed on $\left[t_{0}, t\right) \times \mathbb{R}^{2}$. 
- The estimate of the term $I I_{1}$ : Using the Hölder and Minkowski inequalities, we get

$$
I I_{1}=\left\|(\nabla I u) f^{\prime}(u)\right\|_{L_{t, x}^{\frac{4}{3}}} \lesssim\|\nabla I u\|_{L_{t, x}^{4}}\|u\|_{L_{t, x}^{2 p}}^{p} \lesssim Z_{I}(t)\|u\|_{L_{t, x}^{2 p}}^{p}
$$

It remains to estimate $\|u\|_{L_{t, x}^{2 p}}^{p}$. We decompose $u=u_{\leqslant 1}+u_{1 \leqslant \leqslant N}+u_{>N}$. We estimate the low frequency part by an interpolation and the Bernstein inequality

$$
\begin{aligned}
\left\|u_{\leqslant 1}\right\|_{L_{t, x}^{2 p}}^{p} & \lesssim\left\|u_{\leqslant 1}\right\|_{L_{t, x}^{5}}^{\theta_{1} p}\left\|u_{\leqslant 1}\right\|_{L_{t, x}^{\infty}}^{\left(1-\theta_{1}\right) p} \lesssim \eta^{\theta_{1} p}\left\|u_{\leqslant 1}\right\|_{L_{t}^{\infty} L_{x}^{p+2}}^{\left(1-\theta_{1}\right) p} \\
& \lesssim \eta^{\theta_{1} p} \sup _{s \in\left[t_{0}, t\right]} E(\operatorname{Iu}(s))^{\frac{\left(1-\theta_{1}\right) p}{p+2}}
\end{aligned}
$$

where we have used the condition $p \geqslant \frac{5}{2}$ and $\theta_{1}=\frac{5}{2 p}$.

For the medium frequency part, we use an interpolation, the Sobolev embedding and the Bernstein inequality to estimate

$$
\begin{aligned}
\left\|u_{1 \leqslant \leqslant N}\right\|_{L_{t, x}^{2 p}}^{p} & \lesssim\left\|u_{1 \leqslant \cdot \leqslant N}\right\|_{L_{t, x}^{5}}^{\theta_{2} p}\left\|u_{1 \leqslant \leqslant N}\right\|_{L_{t, x}^{3 p}}^{\left(1-\theta_{2}\right) p} \lesssim \eta^{\theta_{2} p}\left\||\nabla|^{1-\frac{4}{3 p}} u_{1 \leqslant \cdot \leqslant N}\right\|_{L_{t}^{3 p} L_{x}^{\frac{6 p}{3 p-2}}}^{\left(1-\theta_{2}\right) p} \\
& \lesssim \eta^{\theta_{2} p}\left\|\nabla u_{1 \leqslant \cdot \leqslant N}\right\|_{L_{t}^{3 p} L_{x}^{\frac{6 p}{3 p-2}}}^{\left(1-\theta_{2}\right) p} \lesssim \eta^{\theta_{2} p} Z_{I}(t)^{\left(1-\theta_{2}\right) p}
\end{aligned}
$$

where $\theta_{2}=\frac{5}{2(3 p-5)}$ and $\left(3 p, \frac{6 p}{3 p-2}\right) \in \Lambda_{0}$.

For the high frequency part, we use Sobolev embedding and (2.5) with $\sigma=s_{c}$ to obtain

$$
\begin{aligned}
\left\|u_{>N}\right\|_{L_{t, x}^{2 p}}^{p} & \lesssim\left\||\nabla|^{s_{c}} u_{>N}\right\|_{L_{t}^{2 p} L_{x}^{\frac{2 p}{p-1}}}^{p} \lesssim N^{-\left(1-s_{c}\right) p}\|\nabla I u\|_{L_{t}^{2 p} L_{x}^{\frac{2 p}{p-1}}}^{p} \\
& \lesssim N^{-\left(1-s_{c}\right) p} Z_{I}(t)^{p} .
\end{aligned}
$$

Thus, collecting (3.8)-(3.11) yields

$$
\|u\|_{L_{t, x}^{2 p}}^{p} \lesssim g(t)^{p}
$$

and so

$$
I I_{1} \lesssim Z_{I}(t) g(t)^{p}
$$

- The estimate of the term $I I_{2}$ : By the assumption $s>\frac{1+s_{c}}{2}$, we have $\nu:=2 s-s_{c}-1<s$. Thus, we deduce from Lemma 2.3, (3.12) and (2.10)

$$
\begin{aligned}
I I_{2} & =\left\|\nabla I f(u)-(\nabla I u) f^{\prime}(u)\right\|_{L_{t, x}^{\frac{4}{3}}} \\
& \lesssim N^{-\left(s-s_{c}\right)}\|\nabla I u\|_{L_{t, x}^{4}}\left\|\langle\nabla\rangle^{s-s_{c}} f^{\prime}(u)\right\|_{L_{t, x}^{2}} \\
& \lesssim N^{-\left(s-s_{c}\right)} Z_{I}(t)\left(\left\|f^{\prime}(u)\right\|_{L_{t, x}^{2}}+\left\||\nabla|^{s-s_{c}} f^{\prime}(u)\right\|_{L_{t, x}^{2}}\right) \\
& \lesssim N^{-\left(s-s_{c}\right)} Z_{I}(t)\left(\|u\|_{L_{t, x}^{2 p}}^{p}+\|u\|_{L_{t, x}^{2 p}}^{p-1}\left\||\nabla|^{s-s_{c}} u\right\|_{L_{t, x}^{2 p}}\right) \\
& \lesssim N^{-\left(s-s_{c}\right)} Z_{I}(t) g(t)^{p-1}\left[g(t)+\left\||\nabla|^{s-s_{c}} u\right\|_{L_{t, x}^{2 p}}\right] \\
& \lesssim N^{-\left(s-s_{c}\right)} Z_{I}(t) g(t)^{p-1}[g(t)+h(t)]
\end{aligned}
$$


where we have used the same argument as deriving (3.12) to estimate

$$
\begin{aligned}
\left\||\nabla|^{s-s_{c}} u\right\|_{L_{t, x}^{2 p}} & \lesssim\left\|u_{\leqslant 1}\right\|_{L_{t, x}^{2 p}}+\left\||\nabla|^{s-s_{c}} u_{1 \leqslant \leqslant N}\right\|_{L_{t, x}^{2 p}}+\left\||\nabla|^{s-s_{c}} u_{>N}\right\|_{L_{t, x}^{2 p}} \\
& \lesssim\left\|u_{\leqslant 1}\right\|_{L_{t, x}^{2 p}}+\left\|\nabla u_{1 \leqslant \leqslant N}\right\|_{L_{t}^{2 p} L_{x}^{\frac{2 p}{p-1}}}+\left\||\nabla|^{s} u_{>N}\right\|_{L_{t}^{2 p} \frac{2 p}{L_{x}-1}} \\
& \lesssim \eta^{\theta_{1}} \sup _{s \in\left[t_{0}, t\right]} E(I u(s))^{\frac{1-\theta_{1}}{p+2}}+Z_{I}(t)+N^{s-1} Z_{I}(t) \\
& \lesssim h(t) .
\end{aligned}
$$

This estimate together with inequality (3.12) ends the proof of Proposition 3.1

Remark 3.1. We assume the $L_{t, x}^{5}$ interaction Morawetz norm to be small unlike the small interaction Morawetz norm $L_{t}^{4} L_{x}^{8}$ as used in [8]. If we replace (3.3) by

$$
\|u\|_{L_{t}^{4} L_{x}^{8}\left(\left[t_{0}, T\right] \times \mathbb{R}^{2}\right)} \leqslant \eta
$$

then, by the similar argument as above, we need the estimate

$$
\left\|(\nabla I u) f^{\prime}(u)\right\|_{L_{t}^{q_{1}^{\prime}} L_{x}^{r_{1}^{\prime}}} \lesssim\|\nabla I u\|_{L_{t}^{q} L_{x}^{r}}\|u\|_{L_{t}^{\frac{p}{\theta}} L_{x}^{\frac{p}{1}-\theta}}^{p}, \quad\left(q_{1}, r_{1}\right), \quad(q, r) \in \Lambda_{0}, \quad \theta \in[0,1]
$$

together with the low frequency part

$$
\left\|u_{\leqslant 1}\right\|_{L_{t}^{\frac{p}{\theta}} \frac{p}{L_{x}^{\frac{1}{-\theta}}}}^{p} \lesssim\left\|u_{\leqslant 1}\right\|_{L_{t}^{4} L_{x}^{8}}^{4 \theta}\left\|u_{\leqslant 1}\right\|_{L_{t, x}^{\infty}}^{p-4 \theta}
$$

where we need the restriction $\frac{p}{\theta} \geqslant 4$ and $\frac{p}{1-\theta} \geqslant 8$. Therefore,

$$
p \geqslant \min _{0 \leqslant \theta \leqslant 1} \max \{4 \theta, 8(1-\theta)\}=\frac{8}{3} .
$$

This argument, compared with $p \geqslant \frac{5}{2}$ in Proposition 3.1, shows that $L_{t, x}^{5}$ is better than $L_{t}^{4} L_{x}^{8}$.

Next, we show the energy increment of $E(I u)(t)$.

Proposition 3.2 (Energy increment). Let $u(t, x)$ be an $H^{s}$ solution to (1.1) with $f(u)=|u|^{p} u$ defined on $\left[t_{0}, T\right] \times \mathbb{R}^{2}$, which satisfies

$$
\|u\|_{L_{t, x}^{5}\left(\left[t_{0}, T\right] \times \mathbb{R}^{2}\right)} \leqslant \eta
$$

for some small constant $\eta$. Assume $E\left(I u\left(t_{0}\right)\right) \leqslant 1$. Then for $s \geqslant \frac{p}{p+1}, p \geqslant \frac{11}{4}$ and sufficiently large $N$, we have for any $t \in\left[t_{0}, T\right]$

$$
\begin{aligned}
& \quad\left|\sup _{s \in\left[t_{0}, t\right]} E(\operatorname{Iu}(s))-E\left(\operatorname{Iu}\left(t_{0}\right)\right)\right| \\
& \lesssim N^{-\left(1-s_{c}\right)} Z_{I}(t)^{3} g(t)^{p-1}+N^{-\left(s-s_{c}\right)} Z_{I}(t)^{2} g(t)^{p-1}[g(t)+h(t)] \\
& \quad+k(t)\left\{N^{-1} g(t)^{p} Z_{I}(t)+\eta N^{-\left(s-\frac{1}{2}+\frac{1}{q}\right)} m(t)\right\},
\end{aligned}
$$

where $k(t)$ and $m(t)$ are defined by

$$
k(t)=\eta^{\theta_{3}(p+1)} \sup _{s \in\left[t_{0}, t\right]} E(I u(s))^{\frac{\left(1-\theta_{3}\right)(p+1)}{p+2}}+Z_{I}(t)^{p+1}, \theta_{3}=\frac{5}{3(p+1)}
$$

and

$$
m(t)=\eta^{1+\theta_{4}} g(t)^{\left(1-\theta_{4}\right)(p-1)}\left(\eta^{\theta_{4}} g(t)^{1-\theta_{4}}+Z_{I}(t)\right)
$$

with $\theta_{4}=\frac{1}{4 p-10}$ and $q=\frac{5 p}{11}$. 
Proof. Since $i I u_{t}+\Delta I u=I F(u)$, we get by a simple computation

$$
\begin{aligned}
E(I u(t))-E\left(I u\left(t_{0}\right)\right)= & \int_{t_{0}}^{t} \frac{\partial}{\partial s} E(I u(s)) d s \\
= & \operatorname{Re} \int_{t_{0}}^{t} \int_{\mathbb{R}^{d}} \overline{I u_{t}}(-\Delta I u+f(I u)) d x d s \\
= & \operatorname{Re} \int_{t_{0}}^{t} \int_{\mathbb{R}^{d}} \overline{I u_{t}}[f(I u)-I f(u)] d x d s \\
= & -\operatorname{Im} \int_{t_{0}}^{t} \int_{\mathbb{R}^{d}} \overline{\nabla I u} \cdot \nabla[f(I u)-I f(u)] d x d s \\
& -\operatorname{Im} \int_{t_{0}}^{t} \int_{\mathbb{R}^{d}} \overline{I f(u)}[f(I u)-I f(u)] d x d s \\
= & -\operatorname{Im} \int_{t_{0}}^{t} \int_{\mathbb{R}^{d}} \overline{\nabla I u} \cdot \nabla I u\left[f^{\prime}(I u)-f^{\prime}(u)\right] d x d s \\
& -\operatorname{Im} \int_{t_{0}}^{t} \int_{\mathbb{R}^{d}} \overline{\nabla I u} \cdot\left[(\nabla I u) f^{\prime}(u)-I\left(f^{\prime}(u) \nabla u\right)\right] d x d s \\
& -\operatorname{Im} \int_{t_{0}}^{t} \int_{\mathbb{R}^{d}} \overline{I f(u)}[f(I u)-I f(u)] d x d s \\
\triangleq & I I_{1}+I_{2}+I I_{3} .
\end{aligned}
$$

- The estimate of $I I_{1}$ : Since $\left(2 p, \frac{2 p}{p-1}\right) \in \Lambda_{0}$, by inequality (2.5) with $\sigma=s_{c}$, and the Sobolev embedding, we estimate

$$
\begin{aligned}
\left|I I_{1}\right| & \lesssim\|\nabla I u\|_{L_{t, x}^{4}}^{2}\left\|f^{\prime}(I u)-f^{\prime}(u)\right\|_{L_{t, x}^{2}} \\
& \lesssim Z_{I}(t)^{2}\left\|P_{>N} u\right\|_{L_{t, x}^{2 p}}^{2 p}\|u\|_{L_{t, x}^{2 p}}^{p-1} \\
& \lesssim Z_{I}(t)^{2}\left\||\nabla|^{s_{c}} P_{>N} u\right\|_{L_{t}^{2 p} L_{x}^{\frac{2 p}{p-1}} g(t)^{p-1}} \\
& \lesssim Z_{I}(t)^{2} N^{-\left(1-s_{c}\right)}\|\nabla I u\|_{L_{t}^{2 p} L_{x}^{\frac{2 p}{p-1}}} g(t)^{p-1} \\
& \lesssim N^{-\left(1-s_{c}\right)} Z_{I}(t)^{3} g(t)^{p-1} .
\end{aligned}
$$

- The estimate of $I I_{2}$ : Using Hölder's inequality and (3.13), we obtain

$$
\begin{aligned}
\left|I I_{2}\right| & \lesssim\|\nabla I u\|_{L_{t, x}^{4}}\left\|(\nabla I u) f^{\prime}(u)-I\left(f^{\prime}(u) \nabla u\right)\right\|_{L_{t, x}^{\frac{4}{3}}} \\
& \lesssim N^{-\left(s-s_{c}\right)} Z_{I}(t)^{2} g(t)^{p-1}[g(t)+h(t)] .
\end{aligned}
$$

- The estimate of $I I_{3}$ : By Hölder's inequality and Minkowski's inequality, we get

$$
\begin{aligned}
\left|I I_{3}\right| & \lesssim\|I f(u)\|_{L_{t, x}^{4}}\|f(I u)-I f(u)\|_{L_{t, x}^{\frac{4}{3}}} \\
& \lesssim\|u\|_{L_{t, x}^{4(p+1)}}^{p+1}\left\{\left\|I u\left(|I u|^{p}-|u|^{p}\right)\right\|_{L_{t, x}^{\frac{4}{3}}}+\left\|(I u)|u|^{p}-I\left(|u|^{p} u\right)\right\|_{L_{t, x}^{\frac{4}{3}}}\right\} .
\end{aligned}
$$

To estimate $\|u\|_{L_{t, x}^{4(p+1)}}^{p+1}$, we decompose $u=u_{\leqslant 1}+u_{1 \leqslant \leqslant N}+u_{>N}$. Using the same argument as leading to (3.12) and $\left(4(p+1), \frac{4(p+1)}{2 p+1}\right) \in \Lambda_{0}$, one has the inequality by means of (2.5) with 


$$
\begin{aligned}
& \sigma=\frac{p}{p+1} \\
& \|u\|_{L_{t, x}^{4(p+1)}}^{p+1} \\
& \lesssim\left\|u_{\leqslant 1}\right\|_{L_{t, x}^{4(p+1)}}^{p+1}+\left\|u_{1 \leqslant \leqslant N}\right\|_{L_{t, x}^{4(p+1)}}^{p+1}+\left\|u_{>N}\right\|_{L_{t, x}^{4(p+1)}}^{p+1} \\
& \lesssim\|u\|_{L_{t, x}^{5}}^{\theta_{3}(p+1)}\left\|u_{\leqslant 1}\right\|_{L_{t, x}^{\infty}}^{\left(1-\theta_{3}\right)(p+1)}+\left\||\nabla|^{\frac{p}{p+1)}} u_{1 \leqslant \leqslant N}\right\|_{L_{t}^{4(p+1)} L_{x}^{\frac{4(p+1)}{2 p+1}}}^{p+1}+\left\||\nabla|^{\frac{p}{p+1}} u_{\geqslant N}\right\|_{L_{t}^{4(p+1)} L_{x}^{\frac{4(p+1)}{2 p+1}}}^{p+1} \\
& \lesssim \eta^{\theta_{3}(p+1)}\left\|u_{\leqslant 1}\right\|_{L_{t}^{\infty} L_{x}^{p+2}}^{\left(1-\theta_{3}\right)(p+1)}+\left\|\nabla u_{1 \leqslant \leqslant}\right\|_{L_{t}^{4(p+1)} L_{x}^{\frac{4(p+1)}{2 p+1}}}^{p+1}+N^{\left(\frac{p}{p+1}-1\right)(p+1)}\|\nabla I u\|^{p+1}{ }_{L_{t}^{4(p+1)}}^{\frac{4(p+1)}{L_{x}^{2 p+1}}} \\
& \lesssim \eta^{\theta_{3}(p+1)} \sup _{s \in\left[t_{0}, t\right]} E(I u(s))^{\frac{\left(1-\theta_{3}\right)(p+1)}{p+2}}+Z_{I}(t)^{p+1} \\
& \simeq k(t)
\end{aligned}
$$

where $\theta_{3}=\frac{5}{3(p+1)}$ and where we have used the condition $s \geqslant \frac{p}{p+1}$.

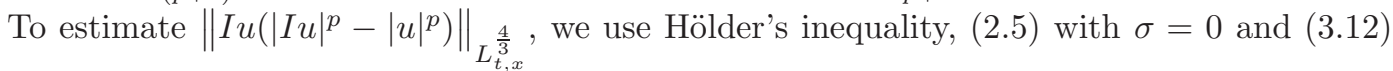
to get

$$
\begin{aligned}
\left\|I u\left(|I u|^{p}-|u|^{p}\right)\right\|_{L_{t, x}^{\frac{4}{3}}} & \lesssim\|I u\|_{L_{t, x}^{2 p}}\|u\|_{L_{t, x}^{2 p}}^{p-1}\|u \geqslant N\|_{L_{t, x}^{4}} \\
& \lesssim\|u\|_{L_{t, x}^{2 p}}^{p} N^{-1}\|\nabla I u\|_{L_{t, x}^{4}} \\
& \lesssim N^{-1} g(t)^{p} Z_{I}(t) .
\end{aligned}
$$

To estimate $\left\|(I u)|u|^{p}-I\left(|u|^{p} u\right)\right\|_{L_{t, x}^{\frac{4}{3}}}$, using (2.9) with $\nu=2 s-\frac{3}{2}+\frac{1}{q} \in(0, s)$ and (2.8), we obtain for $q=\frac{5 p}{11}$

$$
\begin{aligned}
& \left\|(I u)|u|^{p}-I\left(|u|^{p} u\right)\right\|_{L_{t, x}^{\frac{4}{3}}} \\
\lesssim & N^{-\left(s-\frac{1}{2}+\frac{1}{q}\right)}\|I u\|_{L_{t, x}^{5}}\left\|\langle\nabla\rangle^{s-\frac{1}{2}+\frac{1}{q}}|u|^{p}\right\|_{L_{t, x}^{\frac{4 q}{p}}} \\
\lesssim & N^{-\left(s-\frac{1}{2}+\frac{1}{q}\right)} \eta\|u\|_{L_{t, x}^{4 q}}^{p-1}\left(\|u\|_{L_{t, x}^{4 q}}+\left\||\nabla|^{s-\frac{1}{2}+\frac{1}{q}} u\right\|_{L_{t, x}^{4 q}}\right) \\
\lesssim & \eta^{1+\theta_{4}(p-1)} N^{-\left(s-\frac{1}{2}+\frac{1}{q}\right)} g(t)^{\left(1-\theta_{4}\right)(p-1)}\left(\eta^{\theta_{4}} g(t)^{1-\theta_{4}}+Z_{I}(t)\right) \\
\lesssim & N^{-\left(s-\frac{1}{2}+\frac{1}{q}\right)} m(t),
\end{aligned}
$$

where we have used the estimates

$$
\|u\|_{L_{t, x}^{4 q}} \lesssim\|u\|_{L_{t, x}^{5}}^{\theta_{4}}\|u\|_{L_{t, x}^{2 p}}^{1-\theta_{4}} \lesssim \eta^{\theta_{4}} g(t)^{1-\theta_{4}}, \theta_{4}=\frac{1}{4 p-10}
$$

and

$$
\begin{aligned}
\left\||\nabla|^{s-\frac{1}{2}+\frac{1}{q}} u\right\|_{L_{t, x}^{4 q}} & \lesssim\left\|u_{\leqslant 1}\right\|_{L_{t, x}^{4 q}}+\left\||\nabla|^{s} u_{1 \leqslant \leqslant N}\right\|_{L_{t}^{4 q} L_{x}^{r}}+\left\||\nabla|^{s} u_{\geqslant N}\right\|_{L_{t}^{4 q} L_{x}^{r}} \\
& \lesssim \eta^{\theta_{4}} g(t)^{1-\theta_{4}}+Z_{I}(t)+N^{1-s} Z_{I}(t),
\end{aligned}
$$

with $r=\frac{20 p}{5 p-11},(4 q, r) \in \Lambda_{0}$. Since inequality $p \geqslant \frac{11}{4}$ guarantees $4 q \geqslant 5$, the interpolation inequality in (3.24) is valid. Thus, plugging (3.21) - (3.23) into (3.20), we get

$$
\left|I I_{3}\right| \lesssim k(t)\left\{N^{-1} g(t)^{p} Z_{I}(t)+N^{-\left(s-\frac{1}{2}+\frac{1}{q}\right)} m(t)\right\}
$$

This estimate, together with (3.18), (3.19), yields (3.17). 
Now we use a standard bootstrap argument to show that the quantity $E(I u)(t)$ is "almost conserved" by making use of inequality

$$
s-s_{c} \leqslant \min \left\{1-s_{c}, s-\frac{1}{2}+\frac{1}{q}\right\}
$$

Proposition 3.3 (Almost conservation law). Let $u(t, x)$ be an $H^{s}$ solution to problem (1.1) with $f(u)=|u|^{p} u$ defined on $\left[t_{0}, T\right] \times \mathbb{R}^{2}$ and satisfy

$$
\|u\|_{L_{t, x}^{5}\left(\left[t_{0}, T\right] \times \mathbb{R}^{2}\right)} \leqslant \eta
$$

for some small constant $\eta$. Assume $E\left(\operatorname{Iu}\left(t_{0}\right)\right) \leqslant 1$. Then for

$$
s \geqslant \max \left\{\frac{1+s_{c}}{2}, \frac{p}{p+1}\right\}, \quad p \geqslant \frac{11}{4}
$$

and sufficiently large $N$, we have

$$
E(I u)(t)=E\left(I u\left(t_{0}\right)\right)+O\left(N^{s_{c}-s}\right) .
$$

Proof. Expression (3.27) will follow from Proposition 3.1 and 3.2 provided we establish

$$
Z_{I}(t) \lesssim 1 \quad \text { and } \quad \sup _{s \in\left[t_{0}, t\right]} E(I u(s)) \lesssim 1, \quad \forall t \in\left[t_{0}, T\right]
$$

From the assumption $E\left(I u\left(t_{0}\right)\right) \leqslant 1$, we only need to prove that

$$
Z_{I}(t) \lesssim\left\|\nabla I u\left(t_{0}\right)\right\|_{2}, \quad \forall t \in\left[t_{0}, T\right]
$$

and

$$
\sup _{s \in\left[t_{0}, t\right]} E(I u(s)) \lesssim E\left(I u\left(t_{0}\right)\right), \quad \forall t \in\left[t_{0}, T\right] .
$$

We show it by a standard bootstrap argument. It suffices to show that the above two properties hold on the interval $\left[t_{0}, T\right]$. Let

$$
\begin{aligned}
& \Omega_{1}:=\left\{t \in\left[t_{0}, T\right]: Z_{I}(t) \leqslant C_{1}\left\|\nabla I u_{0}\right\|_{2}, \sup _{s \in\left[t_{0}, t\right]} E(I u(s)) \leqslant C_{2} E\left(I u\left(t_{0}\right)\right)\right\}, \\
& \Omega_{2}:=\left\{t \in\left[t_{0}, T\right]: Z_{I}(t) \leqslant 2 C_{1}\left\|\nabla I u_{0}\right\|_{2}, \sup _{s \in\left[t_{0}, t\right]} E(\operatorname{Iu}(s)) \leqslant 2 C_{2} E\left(\operatorname{Iu}\left(t_{0}\right)\right)\right\},
\end{aligned}
$$

where $C_{1}$ and $C_{2}$ are sufficiently large constants which may depend on the Strichartz constant. In order to run the bootstrap argument successfully, we need to verify three properties:

(1) $\Omega_{1}$ is a nonempty closed set.

(2) $\Omega_{2} \subset \Omega_{1}$.

(3) If $t \in \Omega_{1}$, then there exists $\varepsilon>0$ such that $[t, t+\varepsilon) \subset \Omega_{2}$.

In fact, since $t_{0} \in \Omega_{1}$, one easily verifies $\Omega_{1}$ is a nonempty closed by Fatou's Lemma. Combining Proposition 3.1 and 3.2 yields (2) by taking $N$ sufficiently large and $\eta$ sufficiently small depending on $C_{1}, C_{2}$ and $E\left(I u\left(t_{0}\right)\right)$. property (3) follows from (2) and from the local well-posedness theory.

The last two statements show that $\Omega_{1}$ is open from the right-hand side and Proposition 3.3 is proved. 
3.2. Global well-posedness. In this part, we establish the global time-space estimates in terms of a rough norm of initial data by making use of the interaction Morawetz estimate and almost conservation law with a scaling argument.

Proposition 3.4. Suppose $u(t, x)$ is a global solution to problem (1.1) with $f(u)=|u|^{p} u$ satisfying $u_{0} \in C_{0}^{\infty}\left(\mathbb{R}^{2}\right)$. Then for

$$
\max \left\{\frac{p}{p+1}, \frac{1+s_{c}}{2}, s_{1}\right\}<s<1 \text { and } p \geqslant \frac{11}{4},
$$

we have

$$
\begin{aligned}
\|u\|_{L_{t, x}^{5}\left(\mathbb{R} \times \mathbb{R}^{2}\right)} & \leqslant C\left(\left\|u_{0}\right\|_{H^{s}\left(\mathbb{R}^{2}\right)}\right), \\
\sup _{t \in \mathbb{R}}\|u(t)\|_{H^{s}\left(\mathbb{R}^{2}\right)} & \leqslant C\left(\left\|u_{0}\right\|_{H^{s}\left(\mathbb{R}^{2}\right)}\right),
\end{aligned}
$$

where $s_{1}$ is the positive root of the quadratic equation

$$
s^{2}+2 s_{c} s+s_{c}^{2}-4 s_{c}=0 .
$$

Remark 3.2. From the local well-posedness theory, we know that the lifespan of a local solution depends only on the $H^{s}$-norm of the initial data. Thus, the global well-posedness part of Theorem 1.1 follows from (3.32) and the standard density argument.

Proof of Proposition (3.4). If $u$ is a solution to problem (1.1) with $f(u)=|u|^{p} u$, so is

$$
u^{\lambda}(t, x)=\lambda^{-\frac{2}{p}} u\left(\frac{t}{\lambda^{2}}, \frac{x}{\lambda}\right)
$$

By inequality (2.6) and the Sobolev embedding, we have

$$
\begin{aligned}
\left\|\nabla I u_{0}^{\lambda}\right\|_{L^{2}\left(\mathbb{R}^{2}\right)} & \lesssim N^{1-s}\left\|u_{0}^{\lambda}\right\|_{\dot{H}^{s}} \simeq N^{1-s} \lambda^{s_{c}-s}\left\|u_{0}\right\|_{\dot{H}^{s}}, \\
\left\|I u_{0}^{\lambda}\right\|_{L^{p+2}} & \lesssim\left\|u_{0}^{\lambda}\right\|_{L^{p+2}}=\lambda^{-\frac{2}{p}+\frac{2}{p+2}}\left\|u_{0}\right\|_{p+2} \lesssim \lambda^{-\frac{2}{p}+\frac{2}{p+2}}\left\|u_{0}\right\|_{H^{s}} .
\end{aligned}
$$

As $s>s_{c}$, taking $\lambda$ sufficiently large depending on $\left\|u_{0}\right\|_{H^{s}}$ and $N$ such that

$$
N^{1-s} \lambda^{s_{c}-s}\left\|u_{0}\right\|_{\dot{H}^{s}} \ll 1 \text { and } \lambda^{-\frac{2}{p}+\frac{2}{p+2}}\left\|u_{0}\right\|_{H^{s}} \ll 1
$$

we get

$$
E\left(I u_{0}^{\lambda}\right) \ll 1 .
$$

Next, we claim that there exists an absolute constant $C$ such that

$$
\left\|u^{\lambda}\right\|_{L_{t, x}^{5}\left(\mathbb{R} \times \mathbb{R}^{2}\right)} \leqslant C \lambda^{\frac{4}{5} s_{c}} .
$$

Choosing $\lambda=1$ yields (3.31). We prove inequality (3.36) via a bootstrap argument. By time reversal symmetry, it suffices to argue for positive time only. Define

$$
\Omega_{1}:=\left\{t \in[0, \infty):\left\|u^{\lambda}\right\|_{L_{t, x}^{5}\left([0, t] \times \mathbb{R}^{2}\right)} \leqslant C \lambda^{\frac{4}{5} s_{c}}\right\} .
$$

Our goal is to prove $\Omega_{1}=[0, \infty)$. Let

$$
\Omega_{2}:=\left\{t \in[0, \infty):\left\|u^{\lambda}\right\|_{L_{t, x}^{5}\left([0, t] \times \mathbb{R}^{2}\right)} \leqslant 2 C \lambda^{\frac{4}{5} s_{c}}\right\} .
$$

In order to run the bootstrap argument successfully, we need to check the following properities:

(1) $\Omega_{1}$ is a nonempty closed(as $0 \in \Omega_{1}$ and using Fatou's Lemma);

(2) $\Omega_{2} \subset \Omega_{1}$

(3) If $t \in \Omega_{1}$, then there exists $\varepsilon>0$ such that $[t, t+\varepsilon) \subset \Omega_{2}$. 
Property (3) follows from (2) and the local well-posedness theory. Thus, it suffices to prove (2): For any $T \in \Omega_{2}$, we want to show that $T \in \Omega_{1}$. Throughout the following proof, all the space-time norms will be computed on $[0, T] \times \mathbb{R}^{2}$.

Using the interaction Morawetz estimate and the mass conservation, we get

$$
\left\|u^{\lambda}\right\|_{L_{t, x}^{5}} \lesssim\left\|u_{0}^{\lambda}\right\|_{2}^{\frac{3}{5}}\left\|u^{\lambda}\right\|_{L_{t}^{\infty} \dot{H}^{\frac{1}{2}}}^{\frac{2}{5}} \lesssim \lambda^{\frac{3}{5} s_{c}}\left\|u_{0}\right\|_{2}^{\frac{3}{5}}\left\|u^{\lambda}\right\|_{L_{t}^{\infty} \dot{H}^{\frac{1}{2}}}^{\frac{2}{5}}
$$

To control the term $\left\|u^{\lambda}(t)\right\|_{L_{t}^{\infty} \dot{H}^{\frac{1}{2}}}$, we decompose $u^{\lambda}=P_{\leqslant N} u^{\lambda}+P_{>N} u^{\lambda}$.

For the low frequency part, we interpolate between the $L_{x}^{2}$-norm and $\dot{H}^{1}$-norm and we use the fact that the operator $I$ is the identity on frequencies $|\xi| \leqslant N$ :

$$
\left\|P_{\leqslant N} u^{\lambda}\right\|_{\dot{H}^{\frac{1}{2}}} \lesssim\left\|P_{\leqslant N} u^{\lambda}\right\|_{2}^{\frac{1}{2}}\left\|P_{\leqslant N} u^{\lambda}\right\|_{\dot{H}^{1}}^{\frac{1}{2}} \lesssim \lambda^{\frac{1}{2} s_{c}}\left\|u_{0}\right\|_{2}^{\frac{1}{2}}\left\|I u^{\lambda}\right\|_{\dot{H}^{1}}^{\frac{1}{2}}
$$

To estimate the high frequency part, we interpolate between the $L_{x}^{2}$-norm and $\dot{H}^{s}$-norm and use (2.5) and (3.34) to obtain

$$
\begin{aligned}
\left\|P_{>N} u^{\lambda}\right\|_{\dot{H}^{\frac{1}{2}}} & \lesssim\left\|P_{>N} u^{\lambda}\right\|_{2}^{1-\frac{1}{2 s}}\left\|P_{>N} u^{\lambda}\right\|_{\dot{H}^{s}}^{\frac{1}{2 s}} \\
& \lesssim \lambda^{\left(1-\frac{1}{2 s}\right) s_{c}}\left\|u_{0}\right\|_{2}^{1-\frac{1}{2 s}} N^{\frac{s-1}{2 s}}\left\|I u^{\lambda}\right\|_{\dot{H}^{1}}^{\frac{1}{2 s}} \\
& \lesssim \lambda^{s_{c}-\frac{1}{2}}\left\|u_{0}\right\|_{2}^{1-\frac{1}{2 s}}\left\|I u^{\lambda}\right\|_{\dot{H}^{1}}^{\frac{1}{2 s}} .
\end{aligned}
$$

Plugging (3.38) and (3.39) into (3.37), we estimate

$$
\begin{aligned}
\left\|u^{\lambda}\right\|_{L_{t, x}^{5}} & \lesssim \lambda^{\frac{3}{5} s_{c}}\left\|u_{0}\right\|_{2}^{\frac{3}{5}}\left\|u^{\lambda}\right\|_{L_{t}^{\infty} \dot{H}^{\frac{1}{2}}}^{\frac{2}{5}} \\
& \lesssim \lambda^{\frac{3}{5} s_{c}}\left\|u_{0}\right\|_{2}^{\frac{3}{5}} \sup _{s \in[0, T]}\left(\lambda^{\frac{1}{2} s_{c}}\left\|u_{0}\right\|_{2}^{\frac{1}{2}}\left\|I u^{\lambda}\right\|_{\dot{H}^{1}}^{\frac{1}{2}}+\lambda^{s_{c}-\frac{1}{2}}\left\|u_{0}\right\|_{2}^{1-\frac{1}{2 s}}\left\|I u^{\lambda}\right\|_{\dot{H}^{1}}^{\frac{1}{2 s}}\right)^{\frac{2}{5}} \\
& \lesssim C\left(\left\|u_{0}\right\|_{2}\right) \lambda^{\frac{4}{5} s_{c}} \sup _{s \in[0, T]}\left(\left\|I u^{\lambda}\right\|_{\dot{H}^{1}}^{\frac{1}{2}}+\left\|I u^{\lambda}\right\|_{\dot{H}^{1}}^{\frac{1}{2 s}}\right)^{\frac{2}{5}}
\end{aligned}
$$

where we have used the fact $\lambda \gg 1$ in the last inequality. Thus, choosing $C$ sufficiently large depending on $\left\|u_{0}\right\|_{2}$, we obtain $T \in \Omega_{1}$ provided we can prove

$$
\sup _{s \in[0, T]}\left\|I u^{\lambda}\right\|_{\dot{H}^{1}} \leqslant 1, \quad T \in \Omega_{2} .
$$

In fact, let $\eta>0$ be sufficiently small constant as in Proposition 3.3 , and we divide [0,T] into

$$
L \sim\left(\frac{\lambda^{\frac{4}{5} s_{c}}}{\eta}\right)^{5} \sim \lambda^{4 s_{c}},
$$

subintervals $I_{j}=\left[t_{j}, t_{j+1}\right]$ such that

$$
\left\|u^{\lambda}\right\|_{L_{t, x}^{5}\left(I_{j} \times \mathbb{R}^{2}\right)} \leqslant \eta
$$

Using Proposition 3.3 on each interval $I_{j}$, we obtain

$$
\sup _{t \in[0, T]} E\left(I u^{\lambda}\right)(t) \leqslant E\left(I u_{0}^{\lambda}\right)+L N^{s_{c}-s} .
$$

To control the changes of energy during the iteration, we need

$$
L N^{s_{c}-s} \simeq \lambda^{4 s_{c}} N^{s_{c}-s} \ll 1 .
$$

This fact together with (3.34) leads to

$$
N^{4 s_{c} \frac{s-1}{s_{c}-s}} N^{s_{c}-s} \simeq N^{-\frac{s^{2}+2 s_{c} s+s_{c}^{2}-4 s_{c}}{s-s_{c}}} \ll 1 .
$$


This may be ensured by taking $N=N\left(\left\|u_{0}\right\|_{H^{s}}\right)$ large enough provided that $s$ satisfies

$$
s^{2}+2 s_{c} s+s_{c}^{2}-4 s_{c}>0 .
$$

This can be verified by $s>s_{1}$ by the definition of $s_{1}$. This completes the bootstrap argument, hence we prove the claim (3.36), and moreover, (3.31) follows.

To deal with $\|u(t)\|_{H^{s}}$, by the conservation of mass, and inequalities (2.6) and (3.40), we estimate

$$
\begin{aligned}
\|u(t)\|_{H^{s}} & \lesssim\left\|u_{0}\right\|_{L^{2}}+\|u(t)\|_{\dot{H}^{s}} \\
& \lesssim\left\|u_{0}\right\|_{L^{2}}+\lambda^{s-s_{c}}\left\|u^{\lambda}\left(\lambda^{2} t\right)\right\|_{\dot{H}^{s}} \\
& \lesssim\left\|u_{0}\right\|_{L^{2}}+\lambda^{s-s_{c}}\left\|I u^{\lambda}\left(\lambda^{2} t\right)\right\|_{H^{1}} \\
& \lesssim\left\|u_{0}\right\|_{L^{2}}+\lambda^{s-s_{c}}\left(\left\|u_{0}^{\lambda}\right\|_{2}+\left\|I u^{\lambda}\left(\lambda^{2} t\right)\right\|_{\dot{H}^{1}}\right) \\
& \lesssim\left\|u_{0}\right\|_{L^{2}}+\lambda^{s-s_{c}}\left(\lambda^{s_{c}}\left\|u_{0}\right\|_{2}+1\right) \\
& \leqslant C\left(\left\|u_{0}\right\|_{H^{s}}\right) .
\end{aligned}
$$

This completes the proof of (3.32).

3.3. Scattering. We prove that the scattering part of Theorem 1.1 holds for $H_{x}^{s}\left(\mathbb{R}^{2}\right)$ with $s \in\left(s_{0}, 1\right)$. We first show that the global Morawetz estimate can be improved to the global Strichartz estimate

$$
\left\|\langle\nabla\rangle^{s} u\right\|_{S^{0}(\mathbb{R})}:=\sup _{(q, r) \in \Lambda_{0}}\left\|\langle\nabla\rangle^{s} u\right\|_{L_{t}^{q} L_{x}^{r}\left(\mathbb{R} \times \mathbb{R}^{2}\right)} .
$$

Second, we use this estimate to show the asymptotic completeness property. Since the construction of the wave operator is standard, we omit it here.

Let $u$ be a global solution to problem (1.1). From the interaction Morawetz estimate (3.31), we have

$$
\|u\|_{L_{t, x}^{5}\left(\mathbb{R} \times \mathbb{R}^{2}\right)} \leqslant C\left(\left\|u_{0}\right\|_{H^{s}}\right) .
$$

Let $\eta>0$ be a small constant to be chosen later and split $\mathbb{R}$ into $L=L\left(\left\|u_{0}\right\|_{H^{s}}\right)$ subintervals $I_{j}=\left[t_{j}, t_{j+1}\right]$ such that

$$
\|u\|_{L_{t, x}^{5}\left(I_{j} \times \mathbb{R}^{2}\right)} \leqslant \eta
$$

Using (2.8) and (3.32), One gets

$$
\begin{aligned}
\left\|\langle\nabla\rangle^{s} u\right\|_{S^{0}\left(I_{j}\right)} & \lesssim\left\|u\left(t_{j}\right)\right\|_{H^{s}}+\left\|\langle\nabla\rangle^{s}\left(|u|^{p} u\right)\right\|_{L_{t . x}^{\frac{4}{3}}\left(I_{j} \times \mathbb{R}^{2}\right)} \\
& \lesssim C\left(\left\|u_{0}\right\|_{H^{s}}\right)+\|u\|_{L_{t, x}^{2 p}\left(I_{j} \times \mathbb{R}^{2}\right)}^{p}\left\|\langle\nabla\rangle^{s} u\right\|_{L_{t, x}^{4}\left(I_{j} \times \mathbb{R}^{2}\right)} .
\end{aligned}
$$

We use the interpolation and the Sobolev embedding to estimate

$$
\begin{aligned}
\|u\|_{L_{t, x}^{2 p}\left(I_{j} \times \mathbb{R}^{2}\right)}^{p} & \lesssim\|u\|_{L_{t, x}^{5}\left(I_{j} \times \mathbb{R}^{2}\right)}^{\theta_{2} p}\|u\|_{L_{t, x}^{3 p}\left(I_{j} \times \mathbb{R}^{2}\right)}^{\left(1-\theta_{2}\right) p} \\
& \lesssim \eta^{\theta_{2} p}\left\||\nabla|^{1-\frac{4}{3 p}} u\right\|_{L_{t}^{3 p} L_{x}^{\frac{6 p}{3 p-2}}\left(I_{j} \times \mathbb{R}^{2}\right)}^{\left(1-\theta_{2}\right) p} \\
& \lesssim \eta^{\theta_{2} p}\left\|\langle\nabla\rangle^{s} u\right\|_{S^{0}\left(I_{j}\right)}^{\left(1-\theta_{2}\right) p}, \quad \theta_{2}=\frac{5}{2(3 p-5)},
\end{aligned}
$$

where $\left(3 p, \frac{6 p}{3 p-2}\right) \in \Lambda_{0}$, and we have used the fact $s>\frac{1+s_{c}}{2}>1-\frac{4}{3 p}$. Hence,

$$
\left\|\langle\nabla\rangle^{s} u\right\|_{S^{0}\left(I_{j}\right)} \lesssim C\left(\left\|u_{0}\right\|_{H^{s}}\right)+\eta^{\frac{5}{2}}\left\|\langle\nabla\rangle^{s} u\right\|_{S^{0}\left(I_{j}\right)}^{p-\frac{5}{2}} .
$$


By a standard continuity argument, we have

$$
\left\|\langle\nabla\rangle^{s} u\right\|_{S^{0}\left(I_{j}\right)} \leqslant C\left(\left\|u_{0}\right\|_{H^{s}}\right),
$$

provided that we take $\eta$ sufficiently small depending on the initial data $\left\|u_{0}\right\|_{H^{s}}$. Summing over all subintervals $I_{j}$, we obtain

$$
\left\|\langle\nabla\rangle^{s} u\right\|_{S^{0}(\mathbb{R})} \leqslant C\left(\left\|u_{0}\right\|_{H^{s}}\right)
$$

Finally, we utilize this estimate to show the asymptotic completeness. It suffices to prove that there exists a unique $u_{ \pm}$such that

$$
\lim _{t \rightarrow \pm \infty}\left\|u(t)-e^{i t \Delta} u_{ \pm}\right\|_{H_{x}^{s}}=0 .
$$

By time reversal symmetry, we only need to prove it for positive times. For $t>0$, we will show that $v(t):=e^{-i t \Delta} u(t)$ converges in $H_{x}^{s}$ as $t \rightarrow+\infty$, and denote $u_{+}$to be the limit. In fact, we obtain by Duhamel's formula

$$
v(t)=u_{0}-i \int_{0}^{t} e^{-i \tau \Delta} f(u)(\tau) d \tau
$$

Hence, for $0<t_{1}<t_{2}$, we have

$$
v\left(t_{2}\right)-v\left(t_{1}\right)=-i \int_{t_{1}}^{t_{2}} e^{-i \tau \Delta} f(u)(\tau) d \tau
$$

By the Strichartz estimate and (3.48), we deduce that

$$
\begin{aligned}
\left\|v\left(t_{2}\right)-v\left(t_{1}\right)\right\|_{H^{s}\left(\mathbb{R}^{2}\right)} & =\left\|\int_{t_{1}}^{t_{2}} e^{-i \tau \Delta} f(u)(\tau) d \tau\right\|_{H^{s}\left(\mathbb{R}^{2}\right)} \\
& \lesssim\|u\|_{L_{t, x}^{5}\left(\left[t_{1}, t_{2}\right] \times \mathbb{R}^{2}\right)}^{\theta_{2}}\left\|\langle\nabla\rangle^{s} u\right\|_{S^{0}\left(\left[t_{1}, t_{2}\right]\right)}^{1+\left(1-\theta_{2}\right) p} \\
& \rightarrow 0 \quad \text { as } t_{1}, t_{2} \rightarrow \infty .
\end{aligned}
$$

Thus, the limit of (3.51) as $+\infty$ is well defined. In particular, we find that

$$
u_{+}=u_{0}-i \int_{0}^{\infty} e^{-i \tau \Delta} f(u)(\tau) d \tau
$$

is nothing but the asymptotic state. Therefore, we completed the proof of Theorem 1.1

\section{Proof of Theorem 1.2}

In this section, we will use the I-method and the improved interaction Morawetz estimates in Lemma 2.6 to show Theorem 1.2 The process is similar to the one in the preceding section. First, we define

$$
Z_{I}(t):=\|I u\|_{Z(t)}=\sup _{(q, r) \in \Lambda_{0}}\left(\sum_{N \geqslant 1}\left\|\nabla P_{N} I u(t)\right\|_{L_{t}^{q} L_{x}^{r}\left(\left[t_{0}, t\right) \times \mathbb{R}^{2}\right)}^{2}\right)^{\frac{1}{2}}
$$

with the convention that $P_{1}=P_{\leqslant 1}$. Then, we have to control $Z_{I}(t)$ as follows.

Proposition 4.1 (The control of $\left.Z_{I}(t)\right)$. Let $u(t, x)$ be an $H^{s}$ solution to problem (1.1) with $f(u)=|u|^{p} u$ defined on $\left[t_{0}, T\right] \times \mathbb{R}^{2}$ and satisfying

$$
\|u\|_{L_{t, x}^{4}\left(\left[t_{0}, T\right] \times \mathbb{R}^{2}\right)} \leqslant \eta
$$

for some small constant $\eta$. Assume $E\left(I u\left(t_{0}\right)\right) \leqslant 1$. Then for $s>\frac{1+s_{c}}{2}, p \geqslant 2$ and sufficiently large $N$, we have for any $t \in\left[t_{0}, T\right]$

$$
Z_{I}(t) \lesssim\left\|\nabla I u\left(t_{0}\right)\right\|_{2}+g_{1}(t)^{p} Z_{I}(t)+N^{-\left(s-s_{c}\right)} Z_{I}(t) g_{1}(t)^{p-1}\left[g_{1}(t)+h_{1}(t)\right],
$$


where $g_{1}(t)$ and $h_{1}(t)$ are defined by

$$
g_{1}(t)^{p}=\eta^{2} \sup _{s \in\left[t_{0}, t\right]} E(I u(s))^{\frac{2 p-4}{p(p+2)}}+\eta^{\theta p} Z_{I}(t)^{(1-\theta) p}+N^{-\left(1-s_{c}\right) p} Z_{I}(t)^{p}
$$

with $\theta=\frac{2}{3 p-4}$ and

$$
h_{1}(t)=\eta^{\frac{4}{d}} \sup _{s \in\left[t_{0}, t\right]} E(I u(s))^{\frac{2(p-2)}{p(p+2)}}+Z_{I}(t) .
$$

Proof. The proof is similar to the proof of Proposition 3.1. The only difference is that we use $\|u\|_{L_{t, x}^{4}}$ instead of $\|u\|_{L_{t, x}^{5}}$ in estimates (3.9), (3.10) and (3.14). In this way, one can relax the restriction of $p$, and to obtain estimate (4.3) for $p \geqslant 2$.

Next, we show the energy increment of $E(I u)(t)$.

Proposition 4.2 (Energy increment). Let $u(t, x)$ be an $H^{s}$ solution to problem (1.1) with $f(u)=|u|^{p} u$ defined on $\left[t_{0}, T\right] \times \mathbb{R}^{2}$ and satisfying

$$
\|u\|_{L_{t, x}^{4}\left(\left[t_{0}, T\right] \times \mathbb{R}^{2}\right)} \leqslant \eta
$$

for some small constant $\eta$. Assume $E\left(I u\left(t_{0}\right)\right) \leqslant 1$. Then for $s \geqslant \max \left\{\frac{p}{p+1}, \frac{1+s_{c}}{2}\right\}, p \geqslant 2$ and sufficiently large $N$, we have for any $t \in\left[t_{0}, T\right]$

$$
\begin{aligned}
& \quad\left|\sup _{s \in\left[t_{0}, t\right]} E(I u(s))-E\left(I u\left(t_{0}\right)\right)\right| \\
& \lesssim N^{-\left(1-s_{c}\right)} Z_{I}(t)^{3} g_{1}(t)^{p-1}+N^{-\left(s-s_{c}\right)} Z_{I}(t)^{2} g_{1}(t)^{p-1}\left[g_{1}(t)+h_{1}(t)\right] \\
& \quad+k_{1}(t)\left\{N^{-1} g_{1}(t)^{p} Z_{I}(t)+\eta N^{-\left(s-s_{c}\right)} g_{1}(t)^{p-1}\left(g_{1}(t)+h_{1}(t)\right)\right\},
\end{aligned}
$$

where $g_{1}(t), h_{1}(t)$ are defined as in Proposition 4.1. and $k_{1}(t)$ is defined to be

$$
k_{1}(t)=\eta \sup _{s \in\left[t_{0}, t\right]} E(I u(s))^{\frac{p}{p+2}}+Z_{I}(t)^{p+1} .
$$

Proof. The proof is similar to the proof of Proposition 3.2 In fact, we use $\|u\|_{L_{t, x}^{4}}$ instead of $\|u\|_{L_{t, x}^{5}}$ in estimates (3.18), (3.19), 3.21) and (3.22). However, we estimate (3.23) in a different way as follows. By the assumption $s>\frac{1+s_{c}}{2}$, we have $\nu:=2 s-s_{c}-1 \in(0, s)$. We obtain, by the same argument as deriving (3.13), the following estimate

$$
\begin{aligned}
& \left\|(I u)|u|^{p}-I\left(|u|^{p} u\right)\right\|_{L_{t, x}^{\frac{4}{3}}} \\
\lesssim & N^{-\left(s-s_{c}\right)}\|I u\|_{L_{t, x}^{4}}\left\|\langle\nabla\rangle^{s-s_{c}}|u|^{p}\right\|_{L_{t, x}^{2}} \\
\lesssim & N^{-\left(s-s_{c}\right)} \eta\|u\|_{L_{t, x}^{2 p}}^{p-1}\left(\|u\|_{L_{t, x}^{2 p}}+\left\||\nabla|^{s-s_{c}} u\right\|_{L_{t, x}^{2 p}}\right) \\
\lesssim & \eta N^{-\left(s-s_{c}\right)} g_{1}(t)^{p-1}\left(g_{1}(t)+h_{1}(t)\right) .
\end{aligned}
$$

Combining the above two propositions, a standard bootstrap argument and the same argument as in the proof of Proposition 3.3, we can show that the quantity $E(I u)(t)$ is "almost conserved" in the following sense.

Proposition 4.3 (Almost conservation law). Let $u(t, x)$ be an $H^{s}$ solution to problem (1.1) with $f(u)=|u|^{p} u$ defined on $\left[t_{0}, T\right] \times \mathbb{R}^{2}$ and satisfying

$$
\|u\|_{L_{t, x}^{4}\left(\left[t_{0}, T\right] \times \mathbb{R}^{2}\right)} \leqslant \eta
$$


for some small constant $\eta$. Assume $E\left(I u\left(t_{0}\right)\right) \leqslant 1$. Then for $s \geqslant \max \left\{\frac{1+s_{c}}{2}, \frac{p}{p+1}\right\}, p \geqslant 2$ and sufficiently large $N$, we have

$$
E(I u)(t)=E\left(I u\left(t_{0}\right)\right)+O\left(N^{s_{c}-s}\right) .
$$

Now we turn to prove Theorem 1.2

The proof of Theorem 1.2, Assume $u$ is a solution to problem (1.1) with $f(u)=|u|^{p} u$, then so is

$$
u^{\lambda}(t, x)=\lambda^{-\frac{2}{p}} u\left(\frac{t}{\lambda^{2}}, \frac{x}{\lambda}\right)
$$

Choosing a sufficiently large $\lambda$ depending on $\left\|u_{0}\right\|_{H^{s}}$ and $N$ such that

$$
N^{1-s} \lambda^{s_{c}-s}\left\|u_{0}\right\|_{\dot{H}^{s}} \ll 1 \text { and } \lambda^{-\frac{2}{p}+\frac{2}{p+2}}\left\|u_{0}\right\|_{H^{s}} \ll 1,
$$

we get

$$
E\left(I u_{0}^{\lambda}\right)=\frac{1}{2}\left\|\nabla I u_{0}^{\lambda}\right\|_{L^{2}}^{2}+\frac{1}{p+2}\left\|I u_{0}^{\lambda}\right\|_{L^{p+2}}^{p+2} \ll 1 .
$$

Next we claim that for any arbitrary large $T_{0}>0$, there exists an absolute constant $C$ such that

$$
\left\|u^{\lambda}\right\|_{L_{t, x}^{4}\left(\left[0, \lambda^{2} T_{0}\right] \times \mathbb{R}^{2}\right)} \leqslant C \lambda^{s_{c}}\left(\lambda^{2} T_{0}\right)^{\frac{1}{12}} .
$$

We prove this claim by the standard bootstrap argument. Let us define

$$
\Omega_{1}:=\left\{t \in\left[0, \lambda^{2} T_{0}\right]:\left\|u^{\lambda}\right\|_{L_{t, x}^{4}\left([0, t] \times \mathbb{R}^{2}\right)} \leqslant C \lambda^{s_{c}} t^{\frac{1}{12}}\right\} .
$$

We want to show $\Omega_{1}=\left[0, \lambda^{2} T_{0}\right]$. Let

$$
\Omega_{2}:=\left\{t \in\left[0, \lambda^{2} T_{0}\right]:\left\|u^{\lambda}\right\|_{L_{t, x}^{4}\left([0, t] \times \mathbb{R}^{2}\right)} \leqslant 2 C \lambda^{s_{c}} t^{\frac{1}{12}}\right\} .
$$

By the same argument as deriving Proposition 3.4 it suffices to prove that for any $T \in \Omega_{2}$, we have $T \in \Omega_{1}$. Throughout the following proof, all spacetime norms will be computed on $[0, T] \times \mathbb{R}^{2}$.

Using the interaction Morawetz estimate and the mass conservation, we get

$$
\begin{aligned}
\left\|u^{\lambda}\right\|_{L_{t, x}^{4}}^{4} & \lesssim T^{\frac{1}{3}}\left\|u_{0}^{\lambda}\right\|_{2}^{2}\left\|u^{\lambda}\right\|_{L_{t}^{\infty} \dot{H}^{\frac{1}{2}}}^{2}+T^{\frac{1}{3}}\left\|u_{0}^{\lambda}\right\|_{2}^{4} \\
& \lesssim T^{\frac{1}{3}} \lambda^{2 s_{c}}\left\|u_{0}\right\|_{2}^{2}\left\|u^{\lambda}\right\|_{L_{t}^{\infty} \dot{H}^{\frac{1}{2}}}^{2}+T^{\frac{1}{3}} \lambda^{4 s_{c}}\left\|u_{0}\right\|_{2}^{4} .
\end{aligned}
$$

From (3.38) and (3.39), we have the control of $\left\|u^{\lambda}(t)\right\|_{L_{t}^{\infty} \dot{H}^{\frac{1}{2}}}$ as follows

$$
\begin{aligned}
\left\|u^{\lambda}(t)\right\|_{L_{t}^{\infty} \dot{H}^{\frac{1}{2}}} & \lesssim\left\|P_{\leqslant N} u^{\lambda}\right\|_{\dot{H}^{\frac{1}{2}}}+\left\|P_{>N} u^{\lambda}\right\|_{\dot{H}^{\frac{1}{2}}} \\
& \lesssim \lambda^{\frac{1}{2} s_{c}}\left\|u_{0}\right\|_{2}^{\frac{1}{2}}\left\|I u^{\lambda}\right\|_{\dot{H}^{1}}^{\frac{1}{2}}+\lambda^{s_{c}-\frac{1}{2}}\left\|u_{0}\right\|_{2}^{1-\frac{1}{2 s}}\left\|I u^{\lambda}\right\|_{\dot{H}^{1}}^{\frac{1}{2 s}} .
\end{aligned}
$$

Plugging this into (4.15), we estimate

$$
\begin{aligned}
\left\|u^{\lambda}\right\|_{L_{t, x}^{4}}^{4} & \lesssim\left\|u_{0}\right\|_{2} T^{\frac{1}{3}} \lambda^{2 s_{c}}\left\|u^{\lambda}\right\|_{L_{t}^{\infty} \dot{H}^{\frac{1}{2}}}^{2}+T^{\frac{1}{3}} \lambda^{4 s_{c}} \\
& \lesssim\left\|u_{0}\right\|_{2} T^{\frac{1}{3}} \lambda^{3 s_{c}} \sup _{s \in[0, T]}\left(\left\|I u^{\lambda}\right\|_{\dot{H}^{1}}+\lambda^{s_{c}-1}\left\|I u^{\lambda}\right\|_{\dot{H}^{1}}^{\frac{1}{s}}\right)+T^{\frac{1}{3}} \lambda^{4 s_{c}} \\
& \leqslant C\left(\left\|u_{0}\right\|_{2}\right) T^{\frac{1}{3}}\left[\lambda^{3 s_{c}} \sup _{s \in[0, T]}\left(\left\|I u^{\lambda}\right\|_{\dot{H}^{1}}+\left\|I u^{\lambda}\right\|_{\dot{H}^{1}}^{\frac{1}{s}}\right)+\lambda^{4 s_{c}}\right],
\end{aligned}
$$

where we use the fact that $\lambda \gg 1$ in the last inequality. Thus, choosing $C$ sufficiently large depending on $\left\|u_{0}\right\|_{2}$, we obtain $T \in \Omega_{1}$ provided we can deduce

$$
\sup _{s \in[0, T]}\left\|I u^{\lambda}\right\|_{\dot{H}^{1}} \leqslant 1, \quad T \in \Omega_{2} .
$$


In fact, let $\eta>0$ be sufficiently small constant as in Proposition 4.3 , and we divide $[0, T]$ into

$$
L \sim \frac{T^{\frac{1}{3}} \lambda^{4 s_{c}}}{\eta^{4}}
$$

subintervals $I_{j}=\left[t_{j}, t_{j+1}\right]$ such that

$$
\left\|u^{\lambda}\right\|_{L_{t, x}^{4}\left(I_{j} \times \mathbb{R}^{2}\right)} \leqslant \eta .
$$

Using Proposition 4.3 on each interval $I_{j}$, we obtain

$$
\sup _{t \in[0, T]} E\left(I u^{\lambda}\right)(t) \leqslant E\left(I u_{0}^{\lambda}\right)+L N^{s_{c}-s} .
$$

To control small energy during the iteration, we need

$$
L N^{s_{c}-s} \simeq T^{\frac{1}{3}} \lambda^{4 s_{c}} N^{s_{c}-s} \ll 1 .
$$

This property together with (4.12) and $T \leqslant \lambda^{2} T_{0}$ leads to

$$
T_{0}^{\frac{1}{3}} N^{\left(\frac{2}{3}+4 s_{c}\right) \frac{1-s}{s-s_{c}}-\left(s-s_{c}\right)}=T_{0}^{\frac{1}{3}} N^{-\frac{3\left(s-s_{c}\right)^{2}-2\left(1+6 s_{c}\right)(1-s)}{3\left(s-s_{c}\right)}} \ll 1
$$

by choosing $N=N\left(\left\|u_{0}\right\|_{H^{s}}, T_{0}\right)$ large enough provided that $s$ satisfies

$$
3\left(s-s_{c}\right)^{2}-2\left(1+6 s_{c}\right)(1-s)>0,
$$

i.e. $s>\tilde{s}_{1}$, where $\tilde{s}_{1}$ is the positive root of the quadratic equation

$$
3\left(s-s_{c}\right)^{2}-2\left(1+6 s_{c}\right)(1-s)=0 .
$$

This completes the bootstrap argument and hence the claim (4.14).

To estimate $\|u(t)\|_{H^{s}}$, by the conservation of mass, (2.6) and (4.16), we get for $t \in\left[0, T_{0}\right]$

$$
\begin{aligned}
\|u(t)\|_{H^{s}} & \lesssim\left\|u_{0}\right\|_{L^{2}}+\|u(t)\|_{\dot{H}^{s}} \\
& \lesssim\left\|u_{0}\right\|_{L^{2}}+\lambda^{s-s_{c}}\left\|u^{\lambda}\left(\lambda^{2} t\right)\right\|_{\dot{H}^{s}} \\
& \lesssim\left\|u_{0}\right\|_{L^{2}}+\lambda^{s-s_{c}}\left\|I u^{\lambda}\left(\lambda^{2} t\right)\right\|_{H^{1}} \\
& \lesssim\left\|u_{0}\right\|_{L^{2}}+\lambda^{s-s_{c}}\left(\left\|u_{0}^{\lambda}\right\|_{2}+\left\|I u^{\lambda}\left(\lambda^{2} t\right)\right\|_{\dot{H}^{1}}\right) \\
& \lesssim\left\|u_{0}\right\|_{L^{2}}+\lambda^{s-s_{c}}\left(\lambda^{s_{c}}\left\|u_{0}\right\|_{2}+1\right) \\
& \leqslant C\left(\left\|u_{0}\right\|_{H^{s}}\right)\left(1+\lambda^{s}\right) \\
& \leqslant C\left(\left\|u_{0}\right\|_{H^{s}}\right)\left(1+T_{0}\right)^{\frac{1-s}{3\left(s-s_{c}\right)^{2}-2\left(1+6 s_{c}\right)(1-s)}}+
\end{aligned}
$$

where we use the relationship (4.12) and (4.20) in the last inequality. This completes the proof of Theorem 1.2

\section{Proof of Theorem 1.3}

In this section, we consider the Cauchy problem for the nonlinear Schrödinger equation

$$
\left\{\begin{array}{l}
\left(i \partial_{t}+\Delta\right) u=f(u)=|u|^{p} u+|u|^{2 k} u, \quad(t, x) \in \mathbb{R} \times \mathbb{R}^{2}, \\
u(0, x)=u_{0}(x) \in H^{s}\left(\mathbb{R}^{2}\right) .
\end{array}\right.
$$

If $u(t, x)$ is the solution to (5.1), then

$$
u^{\lambda}(t, x)=\lambda^{-\frac{1}{k}} u\left(\frac{t}{\lambda^{2}}, \frac{x}{\lambda}\right)
$$

is the solution to

$$
\left\{\begin{array}{l}
\left(i \partial_{t}+\Delta\right) u^{\lambda}=\lambda^{-2+\frac{p}{k}}\left|u^{\lambda}\right|^{p} u^{\lambda}+\left|u^{\lambda}\right|^{2 k} u^{\lambda} \\
u^{\lambda}(0, x)=\lambda^{-\frac{1}{k}} u_{0}\left(\frac{x}{\lambda}\right)
\end{array}\right.
$$


The energy $E\left(u^{\lambda}\right)$ is defined by

$$
E\left(u^{\lambda}\right)(t)=\frac{1}{2} \int\left|\nabla u^{\lambda}(t, x)\right|^{2} d x+\frac{1}{2(k+1)} \int\left|u^{\lambda}(t, x)\right|^{2(k+1)} d x+\frac{\lambda^{-2+\frac{p}{k}}}{p+2} \int\left|u^{\lambda}(t, x)\right|^{p+2} d x .
$$

For given $u_{0} \in H^{s}\left(\mathbb{R}^{2}\right)$, we have

$$
\begin{array}{r}
\left\|\nabla I u_{0}^{\lambda}\right\|_{2} \leqslant N^{1-s}\left\|u_{0}^{\lambda}\right\|_{\dot{H}^{s}}=N^{1-s} \lambda^{1-\frac{1}{k}-s}\left\|u_{0}\right\|_{\dot{H}^{s}}, \\
\lambda^{-2+\frac{p}{k}}\left\|u_{0}^{\lambda}\right\|_{p+2}^{p+2}=\lambda^{-2+\frac{p}{k}} \lambda^{-\frac{p+2}{k}+2}\left\|u_{0}\right\|_{p+2}^{p+2} \lesssim \lambda^{-\frac{2}{k}}\left\|u_{0}\right\|_{H_{x}^{s}}^{p+2}, \\
\left\|u_{0}^{\lambda}\right\|_{2 k+2}=\lambda^{-\frac{1}{k}+\frac{1}{k+1}}\left\|u_{0}\right\|_{2 k+2} \lesssim \lambda^{-\frac{1}{k}+\frac{1}{k+1}}\left\|u_{0}\right\|_{H_{x}^{s}}
\end{array}
$$

As $s>1-\frac{1}{k}$, choosing $\lambda$ sufficiently large depending on $\left\|u_{0}\right\|_{H^{s}}$ and $N$ such that

$$
N^{1-s} \lambda^{1-\frac{1}{k}-s}\left\|u_{0}\right\|_{\dot{H}^{s}} \ll 1 \text { and } \lambda^{-\frac{1}{k}+\frac{1}{k+1}}\left\|u_{0}\right\|_{H_{x}^{s}} \ll 1
$$

we obtain

$$
E\left(I u_{0}^{\lambda}\right) \leqslant 1
$$

5.1. Almost conservation law. Let us define $Z_{I}(t)$ by

$$
Z_{I}(t):=\|I u\|_{Z(t)}=\sup _{(q, r) \in \Lambda_{0}}\left(\sum_{N \geqslant 1}\left\|\nabla P_{N} I u(t)\right\|_{L_{t}^{q} L_{x}^{r}\left(\left[t_{0}, t\right) \times \mathbb{R}^{2}\right)}^{2}\right)^{\frac{1}{2}} .
$$

Moreover, we denote

$$
s_{c}^{(1)}=1-\frac{2}{p}, s_{c}^{(2)}=1-\frac{1}{k}, s_{c}^{(1)}<s_{c}^{(2)} .
$$

Proposition 5.1. Let $u(t, x)$ be an $H^{s}$ solution to problem (5.2) defined on $\left[t_{0}, T\right] \times \mathbb{R}^{2}$ and satisfying

$$
\|u\|_{L_{t, x}^{5}\left(\left[t_{0}, T\right] \times \mathbb{R}^{2}\right)} \leqslant \eta
$$

for some small constant $\eta$. Assume $E\left(I u\left(t_{0}\right)\right) \leqslant 1$. Then for sufficiently large $N$,

$$
s>\max \left\{s_{c}^{(1)}, \frac{1+s_{c}^{(1)}}{2}, s_{c}^{(2)}\right\}=\max \left\{\frac{1+s_{c}^{(1)}}{2}, s_{c}^{(2)}\right\}, 2 k>p \geqslant \frac{5}{2},
$$

and $k$ is an integer number larger than one,

$$
Z_{I}(t) \lesssim\left\|\nabla I u_{0}\right\|_{2}+\lambda^{-2+\frac{p}{k}}\left[\tilde{g}(t)^{p} Z_{I}(t)+N^{-\left(s-s_{c}\right)} Z_{I}(t) \tilde{g}(t)^{p-1}(\tilde{g}(t)+\tilde{h}(t))\right]+g_{k}(t) Z_{I}(t) .
$$

where $\tilde{g}(t), \tilde{h}(t)$ and $g_{k}(t)$ are defined by

$$
\left\{\begin{array}{l}
\tilde{g}(t)^{p}=\eta^{\theta_{1} p} \lambda^{\left(2-\frac{p}{k}\right) \frac{\left(1-\theta_{1}\right) p}{p+2}} \sup _{s \in\left[t_{0}, t\right]} E(I u(s))^{\frac{\left(1-\theta_{1}\right) p}{p+2}}+\eta^{\theta_{2} p} Z_{I}(t)^{\left(1-\theta_{2}\right) p}+N^{-\left(1-s_{c}^{(1)}\right) p} Z_{I}(t)^{p} \\
\tilde{h}(t)=\eta^{\theta_{1}} \lambda^{\left(2-\frac{p}{k}\right) \frac{1-\theta_{1}}{p+2}} \sup _{s \in\left[t_{0}, t\right]} E(\operatorname{Iu}(s))^{\frac{1-\theta_{1}}{p+2}}+Z_{I}(t) \\
g_{k}(t)=\eta^{2 k \theta_{1}} \sup _{s \in\left[t_{0}, t\right]} E(\operatorname{Iu}(s))^{\frac{\left(1-\theta_{1}\right) k}{k+1}}+\eta^{2 k \theta_{2}} Z_{I}(t)^{2 k\left(1-\theta_{2}\right)}+N^{-2 k\left(1-s_{c}^{(2)}\right)} Z_{I}(t)^{2 k}
\end{array}\right.
$$

with $\theta_{1}, \theta_{2} \in(0,1)$ defined as in Proposition 3.1.

Proof. Using the Strichartz estimate (2.3), we get from (5.2)

$$
\begin{aligned}
Z_{I}(t) & \lesssim\left\|\nabla I u\left(t_{0}\right)\right\|_{2}+\|\nabla I F(u)\|_{L_{t, x}^{\frac{4}{3}}} \\
& \lesssim\left\|\nabla I u\left(t_{0}\right)\right\|_{2}+\lambda^{-2+\frac{p}{k}}\left\|\nabla I\left(|u|^{p} u\right)\right\|_{L_{t, x}^{\frac{4}{3}}}+\left\|\nabla I\left(|u|^{2 k} u\right)\right\|_{L_{t, x}^{\frac{4}{3}}},
\end{aligned}
$$


where all space-time norms are computed on $\left[t_{0}, t\right) \times \mathbb{R}^{2}$. Using the following estimate of low frequency part

$$
\begin{aligned}
\left\|u_{\leqslant 1}\right\|_{L_{t, x}^{2 p}}^{p} & \lesssim\left\|u_{\leqslant 1}\right\|_{L_{t, x}^{5}}^{\theta_{1} p}\left\|u_{\leqslant 1}\right\|_{L_{t, x}^{\infty}}^{\left(1-\theta_{1}\right) p} \lesssim \eta^{\theta_{1} p}\left\|u_{\leqslant 1}\right\|_{L_{t}^{\infty} L_{x}^{p+2}}^{\left(1-\theta_{1}\right) p} \\
& \lesssim \eta^{\theta_{1} p} \lambda^{\left(2-\frac{p}{k}\right) \frac{\left(1-\theta_{1}\right) p}{p+2}} \sup _{s \in\left[t_{0}, t\right]} E(I u(s))^{\frac{\left(1-\theta_{1}\right) p}{p+2}}
\end{aligned}
$$

we obtain in the same way as deriving (3.8) and (3.13), the inequality

$$
\left\|\nabla I\left(|u|^{p} u\right)\right\|_{L_{t, x}^{\frac{4}{3}}} \lesssim \tilde{g}(t)^{p} Z_{I}(t)+N^{-\left(s-s_{c}\right)} Z_{I}(t) \tilde{g}(t)^{p-1}(\tilde{g}(t)+\tilde{h}(t))
$$

where $\theta_{1}=\frac{5}{2 p}$ with $p \geqslant \frac{5}{2}$.

On the other hand, using the fact that $\nabla I$ acts as a derivative, we obtain

$$
\left\|\nabla I\left(|u|^{2 k} u\right)\right\|_{L_{t, x}^{\frac{4}{3}}} \lesssim\|u\|_{L_{t, x}^{4 k}}^{2 k}\|\nabla I u\|_{L_{t, x}^{4}}, \quad \forall t \in\left[t_{0}, T\right]
$$

Using estimate (3.12) with $p=2 k$, we deduce

$$
\|u\|_{L_{t, x}^{4 k}}^{2 k} \lesssim \eta^{2 k \theta_{1}} \sup _{s \in\left[t_{0}, t\right]} E(I u(s))^{\frac{\left(1-\theta_{1}\right) k}{k+1}}+\eta^{2 k \theta_{2}} Z_{I}(t)^{2 k\left(1-\theta_{2}\right)}+N^{-2} Z_{I}(t)^{2 k}
$$

This together with (5.8), (5.10) and (5.11) yields (5.7).

Proposition 5.2 (Energy increment). Let $u(t, x)$ be an $H^{s}$ solution to problem (5.2) defined on $\left[t_{0}, T\right] \times \mathbb{R}^{2}$ and satisfying

$$
\|u\|_{L_{t, x}^{5}\left([0, T] \times \mathbb{R}^{2}\right)} \leqslant \eta
$$

for some small constant $\eta$. Assume $E\left(I u\left(t_{0}\right)\right) \leqslant 1$. Then for

$$
s \geqslant \frac{2 k}{2 k+1}, \quad 2 k>p \geqslant \frac{11}{4}, \quad 2 \leqslant k \in \mathbb{N}
$$

and sufficiently large $N$, we have

$$
\left|\sup _{s \in\left[t_{0}, t\right]} E(I u(s))-E\left(I u\left(t_{0}\right)\right)\right| \lesssim h_{1}(t)+h_{2}(t)+h_{3}(t)+h_{4}(t)
$$

where the quantities $h_{j}(t)(j=1,2,3,4)$ are defined below in (5.20), (5.21), (5.23), (5.24).

Proof. Since

$$
i I u_{t}+\Delta I u=I f(u)=\lambda^{-2+\frac{p}{k}} I\left(|u|^{p} u\right)+I\left(|u|^{2 k} u\right),
$$


by a simple computation, we obtain

$$
\begin{aligned}
& E(I u(t))-E\left(I u\left(t_{0}\right)\right)=\int_{t_{0}}^{t} \frac{\partial}{\partial s} E(I u(s)) d s \\
& =\operatorname{Im} \int_{t_{0}}^{t} \int_{\mathbb{R}^{d}} \overline{\Delta I u}\left[|I u|^{2 k} I u-I\left(|u|^{2 k} u\right)\right] d x d s \\
& -\operatorname{Im} \int_{t_{0}}^{t} \int_{\mathbb{R}^{d}} \overline{I\left(|u|^{2 k} u\right)}\left[|I u|^{2 k} I u-I\left(|u|^{2 k} u\right)\right] d x d s \\
& -\lambda^{-2+\frac{p}{k}} \operatorname{Im} \int_{t_{0}}^{t} \int_{\mathbb{R}^{d}} \overline{\nabla I u} \cdot \nabla\left[|I u|^{p} I u-I\left(|u|^{p} u\right)\right] d x d s \\
& -\lambda^{-4+\frac{2 p}{k}} \operatorname{Im} \int_{t_{0}}^{t} \int_{\mathbb{R}^{d}} \overline{I\left(|u|^{p} u\right)}\left[|I u|^{p} I u-I\left(|u|^{p} u\right)\right] d x d s \\
& -\lambda^{-2+\frac{p}{k}} \operatorname{Im} \int_{t_{0}}^{t} \int_{\mathbb{R}^{d}} \overline{I\left(|u|^{p} u\right)}\left[|I u|^{2 k} I u-I\left(|u|^{2 k} u\right)\right] d x d s \\
& -\lambda^{-2+\frac{p}{k}} \operatorname{Im} \int_{t_{0}}^{t} \int_{\mathbb{R}^{d}} \overline{I\left(|u|^{2 k} u\right)}\left[|I u|^{p} I u-I\left(|u|^{p} u\right)\right] d x d s .
\end{aligned}
$$

Recalling the result in [ [8], Proposition 5.2], we have for $s>\frac{2(k-1)}{2 k-1}$

$$
\begin{aligned}
& |(5.14)+(5.15)| \\
& \lesssim N^{-1+}\left(Z_{I}(t)^{2 k+2}+\eta^{2 k \theta_{5}} Z_{I}(t)^{2} \sup _{s \in\left[t_{0}, t\right]} E(I u(s))^{\frac{\left(1-\theta_{5}\right) k}{k+1}}\right. \\
& \left.+\sum_{J=3}^{2 k+2} \eta^{(2 k+2-J) \theta_{6}} Z_{I}(t)^{J} \sup _{s \in\left[t_{0}, t\right]} E(I u(s))^{\frac{\left(1-\theta_{6}\right)(2 k+2-J)}{2(k+1)}}\right) \\
& +N^{-1+}\left(Z_{I}(t)^{2 k+1}+\eta^{2 k \theta_{5}} Z_{I}(t) \sup _{s \in\left[t_{0}, t\right]} E(I u(s))^{\frac{\left(1-\theta_{5}\right) k}{k+1}}\right) \\
& \times\left(Z_{I}(t)^{2 k+1}+\eta^{\theta_{7}(2 k+1)} \sup _{s \in\left[t_{0}, t\right]} E(I u(s))^{\frac{\left(1-\theta_{7}\right)(2 k+1)}{2(k+1)}}\right) \\
& +N^{-1}+\sum_{J=3}^{2 k+2} \eta^{(2 k+2-J) \theta_{6}} Z_{I}(t)^{J-1} \sup _{s \in\left[t_{0}, t\right]} E(I u(s))^{\frac{\left(1-\theta_{6}\right)(2 k+2-J)}{2(k+1)}} \\
& \times\left(Z_{I}(t)^{2 k+1}+\eta^{\theta_{7}(2 k+1)} \sup _{s \in\left[t_{0}, t\right]} E(I u(s))^{\frac{\left(1-\theta_{7}\right)(2 k+1)}{2(k+1)}}\right) \\
& =: h_{1}(t) \text {, }
\end{aligned}
$$

where $\theta_{5}, \theta_{6}, \theta_{7} \in(0,1)$ are defined by

$$
\theta_{5}=\frac{5}{4 k}, \theta_{6}=\frac{5}{4(2 k-1)}, \theta_{7}=\frac{5}{3(2 k+1)} .
$$

Here, we adopt the interaction Morawetz norm $L_{t, x}^{5}$ instead of $L_{t}^{4} L_{x}^{8}$-norm as used in [8]. There is only one difference appearing in the power of $\eta$ and $E(I u)$.

While, by Proposition 3.2, we have for $s \geqslant \frac{p}{p+1}$ and $p \geqslant \frac{11}{4}$

$$
\begin{aligned}
& |\underline{(5.16)}+(\underline{5.17})| \\
& \lesssim \lambda^{-2+\frac{p}{k}}\left(N^{-\left(1-s_{c}^{(1)}\right)} Z_{I}(t)^{3} \tilde{g}(t)^{p-1}+N^{-\left(s-s_{c}^{(1)}\right)} Z_{I}(t)^{2} \tilde{g}(t)^{p-1}[\tilde{g}(t)+\tilde{h}(t)]\right) \\
& \quad+\lambda^{-4+\frac{2 p}{k}} \tilde{k}(t)\left(N^{-1} \tilde{g}(t)^{p} Z_{I}(t)+\eta N^{-\left(s-s_{c}^{(1)}+\frac{1}{q}\right)} \tilde{m}(t)\right)=: h_{2}(t),
\end{aligned}
$$


where $q=\frac{5 p}{11}$, and $\tilde{k}(t), \tilde{m}(t)$ are defined by

$$
\left\{\begin{array}{l}
\tilde{k}(t)=\eta^{\theta_{3}(p+1)} \lambda^{\left(2-\frac{p}{k}\right) \frac{\left(1-\theta_{3}\right)(p+1)}{p+2}} \sup _{s \in\left[t_{0}, t\right]} E(I u(s))^{\frac{\left(1-\theta_{3}\right)(p+1)}{p+2}}+Z_{I}(t)^{p+1}, \theta_{3}=\frac{5}{3(p+1)} \\
\tilde{m}(t)=\eta^{1+\theta_{4}} \tilde{g}(t)^{\left(1-\theta_{4}\right)(p-1)}\left(\eta^{\theta_{4}} \tilde{g}(t)^{1-\theta_{4}}+Z_{I}(t)\right), \theta_{4}=\frac{1}{4 p-10} .
\end{array}\right.
$$

- The estimate of (5.18): By the same argument as leading to (5.14), we have for

$$
\begin{aligned}
|\underline{(5.18)}| \lesssim & \lambda^{-2+\frac{p}{k}} N^{-1+} \sup _{N}\left\|P_{N} I\left(|u|^{p} u\right)\right\|_{L_{t, x}^{4}}\left\{Z_{I}(t)^{2 k+2}+\eta^{2 k \theta_{5}} Z_{I}(t)^{2} \sup _{s \in\left[t_{0}, t\right]} E(I u(s))^{\frac{\left(1-\theta_{5}\right) k}{k+1}}\right. \\
& \left.+\sum_{J=3}^{2 k+2} \eta^{(2 k+2-J) \theta_{6}} Z_{I}(t)^{J} \sup _{s \in\left[t_{0}, t\right]} E(I u(s))^{\frac{\left(1-\theta_{6}\right)(2 k+2-J)}{2(k+1)}}\right\}, \quad s>\frac{2(k-1)}{2 k-1}
\end{aligned}
$$

To estimate $\left\|P_{N} I\left(|u|^{p} u\right)\right\|_{L_{t, x}^{4}}$, we obtain by (3.21)

$$
\begin{aligned}
\left\|P_{N} I\left(|u|^{p} u\right)\right\|_{L_{t, x}^{4}} & \lesssim\|u\|_{L_{t, x}^{4(p+1)}}^{p+1} \\
& \lesssim \eta^{\theta_{3}(p+1)} \lambda^{\left(2-\frac{p}{k}\right) \frac{\left(1-\theta_{3}\right)(p+1)}{p+2}} \sup _{s \in\left[t_{0}, t\right]} E(I u(s))^{\frac{\left(1-\theta_{3}\right)(p+1)}{p+2}}+Z_{I}(t)^{p+1},
\end{aligned}
$$

where $\theta_{3}=\frac{5}{3(p+1)}$ and we need the restriction $s \geqslant \frac{p}{p+1}$ in the estimate of the high frequency part by means of (2.5) with $\sigma=\frac{p}{p+1}$. Plugging this into (5.22) gives

|(5.18) |

$$
\begin{aligned}
& \lesssim \lambda^{-2+\frac{p}{k}} N^{-1+}\left(\eta^{\theta_{3}(p+1)} \lambda^{\left(2-\frac{p}{k}\right) \frac{\left(1-\theta_{3}\right)(p+1)}{p+2}} \sup _{s \in\left[t_{0}, t\right]} E(I u(s))^{\frac{\left(1-\theta_{3}\right)(p+1)}{p+2}}+Z_{I}(t)^{p+1}\right) \\
& \times\left\{Z_{I}(t)^{2 k+2}+\eta^{2 k \theta_{5}} Z_{I}(t)^{2} \sup _{s \in\left[t_{0}, t\right]} E(I u(s))^{\frac{\left(1-\theta_{5}\right) k}{k+1}}\right. \\
& \left.+\sum_{J=3}^{2 k+2} \eta^{(2 k+2-J) \theta_{6}} Z_{I}(t)^{J} \sup _{s \in[0, t]} E(I u(s))^{\left(1-\theta_{6}\right)(2 k+2-J)}\right\} \\
& =: h_{3}(t) .
\end{aligned}
$$

- The estimate of (5.19): By the same argument as deducing (3.20), we get

$$
\begin{aligned}
& |\underline{15.19)}| \\
\lesssim & \lambda^{-2+\frac{p}{k}}\|u\|_{L_{t, x}^{4(2 k+1)}}^{2 k+1}\left[\left\|I u\left(|I u|^{p}-|u|^{p}\right)\right\|_{L_{t, x}^{\frac{4}{3}}}+\left\|(I u)|u|^{p}-I\left(|u|^{p} u\right)\right\|_{L_{t, x}^{\frac{4}{3}}}\right] \\
\lesssim & \lambda^{-2+\frac{p}{k}}\left\{\eta^{\theta_{3}(2 k+1)} \sup _{s \in\left[t_{0}, t\right]} E(\operatorname{Iu}(s))^{\frac{\left(1-\theta_{3}\right)(2 k+1)}{2(k+1)}}+Z_{I}(t)^{2 k+1}\right\} \\
& \times\left\{N^{-1} \tilde{g}(t)^{p} Z_{I}(t)+N^{-\left(s-\frac{1}{2}+\frac{1}{q}\right)} \tilde{m}(t)\right\} \\
\simeq & h_{4}(t),
\end{aligned}
$$

where $q=\frac{5 p}{11}, \tilde{g}(t)$ is defined as in Proposition [5.1. We need the restriction $s \geqslant \frac{2 k}{2 k+1}$ in the estimate of the high frequency part by means of (2.5) with $\sigma=\frac{2 k}{2 k+1}$.

Note that $\frac{d k}{2 k+1}>\max \left\{\frac{2(k-1)}{2 k-1}, \frac{p}{p+1}\right\}$ and collecting (5.20)-(5.24), we obtain (5.13). Therefore, we conclude the proof of this proposition. 
Combining the above two propositions, a standard bootstrap argument and arguing as in the proof of Proposition 3.3 we can easily show that the quantity $E(I u)(t)$ is "almost conserved" by using the condition

$$
s_{c}^{(2)}<\max \left\{\frac{1+s_{c}^{(1)}}{2}, \frac{d k}{2 k+1}\right\} .
$$

Proposition 5.3 (Almost conservation law). Let $u(t, x)$ be an $H^{s}$ solution to problem (5.2) with defined on $[0, T] \times \mathbb{R}^{2}$ and satisfying

$$
\|u\|_{L_{t, x}^{5}\left([0, T] \times \mathbb{R}^{2}\right)} \leqslant \eta
$$

for some small constant $\eta$. Assume $E\left(I u_{0}\right) \leqslant 1$. Then for

$$
s \geqslant \max \left\{\frac{1+s_{c}^{(1)}}{2}, \frac{2 k}{2 k+1}\right\}, 2 k>p \geqslant \frac{11}{4}, 2 \leqslant k \in \mathbb{N}
$$

and sufficiently large $N$, we have

$$
E(I u)(t)=E\left(I u_{0}\right)+O\left(\max \left\{N^{-1+}, \lambda^{-\left(2-\frac{p}{k}\right)\left(1-\frac{\left(1-\theta_{1}\right) p}{p+2}\right)} N^{-\left(s-s_{c}^{(1)}\right)}\right\}\right),
$$

where $\theta_{1}=\frac{5}{2 p}$.

Proof. By the same way as deducing Proposition 3.3. we derive that the contributions of $h_{j}(t)(j=1,2,3,4)$ to the difference $E(I u(t))-E\left(I u\left(t_{0}\right)\right)$ are

$$
\begin{aligned}
& N^{-1}, \lambda^{-\left(2-\frac{p}{k}\right)\left(1-\frac{\left(1-\theta_{1}\right) p}{p+2}\right)} N^{-\left(s-s_{c}^{(1)}\right)}, \lambda^{-\left(2-\frac{p}{k}\right)} N^{-1+}, \\
& \lambda^{-\left(2-\frac{p}{k}\right)} N^{-\left(s-\frac{1}{2}+\frac{1}{q}\right)} \lambda^{\left(2-\frac{p}{k}\right)\left(1-\theta_{1}\right)\left(1-\theta_{4}\right) \frac{p}{p+2}}
\end{aligned}
$$

respectively. This fact gives the formula (5.26).

5.2. Global well-posedness and scattering. By an argument as similar to that in Section 3 , we can reduce the proof of Theorem 1.3 to the following proposition.

Proposition 5.4. Suppose $u(t, x)$ is a global solution to problem (1.1) with $f(u)=|u|^{p} u+|u|^{2 k} u$ and $u_{0} \in C_{0}^{\infty}\left(\mathbb{R}^{2}\right)$. Then for

$$
2 k>p \geqslant \frac{11}{4}, 1<k \in \mathbb{N}
$$

and

$$
s \in\left(\tilde{s}_{3}, 1\right), \tilde{s}_{3}:=\max \left\{\frac{1+s_{c}^{(1)}}{2}, \frac{2 k}{2 k+1}, \frac{5 s_{c}^{(2)}}{4 s_{c}^{(2)}+1}, s_{3}\right\}
$$

with $s_{3}$ being the positive root of the quadratic equation

$$
s^{2}-\left(s_{c}^{(1)}+s_{c}^{(2)}-\alpha\right) s-\alpha=0, \alpha=4 s_{c}^{(2)}-\frac{9\left(2-\frac{p}{k}\right)}{2(p+2)},
$$

we have

$$
\begin{aligned}
\|u\|_{L_{t, x}^{5}\left(\mathbb{R} \times \mathbb{R}^{2}\right)} & \leqslant C\left(\left\|u_{0}\right\|_{H^{s}\left(\mathbb{R}^{2}\right)}\right), \\
\sup _{t \in \mathbb{R}}\|u(t)\|_{H^{s}\left(\mathbb{R}^{2}\right)} & \leqslant C\left(\left\|u_{0}\right\|_{H^{s}\left(\mathbb{R}^{2}\right)}\right) .
\end{aligned}
$$

Proof. By the same argument as in the proof of Proposition 3.4 and the scaling transform, we claim that

$$
\left\|u^{\lambda}\right\|_{L_{t, x}^{5}\left(\mathbb{R} \times \mathbb{R}^{2}\right)} \leqslant C \lambda^{\frac{4}{5} s_{c}^{(2)}}
$$

Indeed, we define

$$
\Omega_{1}:=\left\{t \in[0, \infty):\left\|u^{\lambda}\right\|_{L_{t, x}^{5}\left([0, t] \times \mathbb{R}^{2}\right)} \leqslant C \lambda^{\frac{4}{5} s_{c}^{(2)}}\right\} .
$$

We want to show $\Omega_{1}=[0, \infty)$. Let

$$
\Omega_{2}:=\left\{t \in[0, \infty):\left\|u^{\lambda}\right\|_{L_{t, x}^{5}\left([0, t] \times \mathbb{R}^{2}\right)} \leqslant 2 C \lambda^{\frac{4}{5} s_{c}^{(2)}}\right\} .
$$


Then, it suffices to show $\Omega_{2} \subset \Omega_{1}$ by the standard bootstrap argument. In the same way as deriving (3.36), it is sufficient to prove

$$
\sup _{s \in[0, T]}\left\|I u^{\lambda}\right\|_{\dot{H}^{1}} \leqslant 1, \quad T \in \Omega_{2} .
$$

In fact, let $\eta>0$ be a sufficiently small constant as in Proposition 3.3 , and we divide [0,T] into

$$
L \sim\left(\frac{\lambda^{\frac{4}{5} s_{c}^{(2)}}}{\eta}\right)^{5} \sim \lambda^{4 s_{c}^{(2)}}
$$

subintervals $I_{j}=\left[t_{j}, t_{j+1}\right]$ such that

$$
\left\|u^{\lambda}\right\|_{L_{t, x}^{5}\left(I_{j} \times \mathbb{R}^{2}\right)} \leqslant \eta .
$$

Using Proposition 5.3 on each interval $I_{j}$, we obtain

$$
\sup _{t \in[0, T]} E\left(I u^{\lambda}\right)(t) \leqslant E\left(I u_{0}^{\lambda}\right)+L \mathcal{O}\left(\max \left\{N^{-1+}, \lambda^{-\left(2-\frac{p}{k}\right)\left(1-\frac{\left(1-\theta_{1}\right) p}{p+2}\right)} N^{-\left(s-s_{c}^{(1)}\right)}\right\}\right) .
$$

To maintain a small energy during the iteration, we need the estimate

$$
\begin{aligned}
& L \mathcal{O}\left(\max \left\{N^{-1+}, \lambda^{-\left(2-\frac{p}{k}\right)\left(1-\frac{\left(1-\theta_{1}\right) p}{p+2}\right)} N^{-\left(s-s_{c}^{(1)}\right)}\right\}\right) \\
\simeq & \lambda^{4 s_{c}^{(2)}} \mathcal{O}\left(\max \left\{N^{-1+}, \lambda^{-\left(2-\frac{p}{k}\right)\left(1-\frac{\left(1-\theta_{1}\right) p}{p+2}\right)} N^{-\left(s-s_{c}^{(1)}\right)}\right\}\right) \ll 1,
\end{aligned}
$$

which together with (5.3) leads to

$$
\lambda^{4 s_{c}^{(2)}} N^{-1+} \simeq N^{4 s_{c}^{(2)} \frac{1-s}{s-s_{c}^{(2)}}} N^{-1+} \simeq N^{\frac{\left(3 p-4-\frac{4}{p}\right)-s(3 p-4)}{p\left(s+\frac{2}{p}-1\right)-}} \ll 1
$$

and

with

$$
\lambda^{4 s_{c}^{(2)}} \lambda^{-\left(2-\frac{p}{k}\right)\left(1-\frac{\left(1-\theta_{1}\right) p}{p+2}\right)} N^{-\left(s-s_{c}^{(1)}\right)} \simeq N^{\frac{(1-s) \alpha}{s-s_{c}^{(2)}}-\left(s-s_{c}^{(1)}\right)} \ll 1
$$

$$
\alpha=4 s_{c}^{(2)}-\left(2-\frac{p}{k}\right)\left(1-\frac{\left(1-\theta_{1}\right) p}{p+2}\right)=4 s_{c}^{(2)}-\frac{9\left(2-\frac{p}{k}\right)}{2(p+2)} .
$$

They may be ensured by choosing $N=N\left(\left\|u_{0}\right\|_{H^{s}}\right)$ large enough provided

$$
s>\max \left\{\frac{5 s_{c}^{(2)}}{4 s_{c}^{(2)}+1}, s_{3}\right\}
$$

where $s_{3}$ is the positive root of the quadratic equation

$$
s^{2}-\left(s_{c}^{(1)}+s_{c}^{(2)}-\alpha\right) s-\alpha=0 .
$$

This completes the bootstrap argument, and hence Proposition 5.4 follows. Therefore, we conclude Theorem 1.3 .

\section{APPENDIX}

In this Appendix, we state the result in the one dimension. In fact, the proof is the same as in the case dimension two. We utilize the following classical interaction Morawetz estimates in 9,36 ,

$$
\|u\|_{L_{t, x}^{8}(I \times \mathbb{R})} \lesssim\|u\|_{L_{t}^{\infty}\left(I ; \dot{H}^{\frac{1}{2}}(\mathbb{R})\right)}^{\frac{1}{4}}\left\|u_{0}\right\|_{2}^{\frac{3}{4}}
$$

and the improved interaction Morawetz estimates in [7]

$$
\int_{0}^{T} \int_{\mathbb{R}}|u(t, x)|^{6} d x d t \lesssim T^{\frac{1}{3}}\left\|u_{0}\right\|_{L_{x}^{2}}^{4}\|u\|_{L_{t}^{\infty}\left([0, T], \dot{H}_{x}^{1 / 2}\right)}^{2}+T^{\frac{1}{3}}\left\|u_{0}\right\|_{L_{x}^{2}}^{6},
$$

instead of (1.17) and (1.16). 
Define

$$
\begin{aligned}
& s_{0}:=\max \left\{\frac{1+s_{c}}{2}, \frac{p}{2(p+1)}, s_{1}\right\}, s_{c}=\frac{1}{2}-\frac{2}{p}, \\
& \tilde{s}_{0}:=\max \left\{\frac{1+s_{c}}{2}, \frac{d p}{2(p+1)}, \tilde{s}_{1}\right\},
\end{aligned}
$$

where $s_{1}$ is the positive root of the quadratic equation

$$
s^{2}+5 s_{c} s+s_{c}^{2}-7 s_{c}=0,
$$

and $\tilde{s}_{1}$ is the positive root of the quadratic equation

$$
3\left(s-s_{c}\right)^{2}-2\left(1+9 s_{c}\right)(1-s)=0 .
$$

Theorem A.1. (i) Assume that $u_{0} \in H^{s}(\mathbb{R})$ with $s \in\left(s_{0}, 1\right)$ and $p \geqslant \frac{17}{3}$. Then the solution $u$ to $i u_{t}+\Delta u=|u|^{p} u$ is global and scatters.

(ii) Assume that $u_{0} \in H^{s}(\mathbb{R})$ with $s \in\left(\tilde{s}_{0}, 1\right)$ and $p \geqslant 4$. Then the solution $u$ to $i u_{t}+\Delta u=$ $|u|^{p} u$ is global. Furthermore, for any $T>0$,

$$
\sup _{t \in[0, T]}\|u(t)\|_{H^{s}(\mathbb{R})} \leqslant C\left(\left\|u_{0}\right\|_{H^{s}(\mathbb{R})}\right)(1+T)^{\frac{1-s}{3\left(s-s_{c}\right)^{2}-2\left(1+9 s_{c}\right)(1-s)}+} .
$$

(iii) Assume that $u_{0} \in H^{s}(\mathbb{R})$ with

$$
s \in\left(\max \left\{\frac{1+s_{c}^{(2)}}{2}, \frac{p_{2}}{2\left(p_{2}+1\right)}, s_{2}\right\}, 1\right), s_{c}^{(j)}=\frac{1}{2}-\frac{2}{p_{j}}, j=1,2, \frac{17}{3} \leqslant p_{1}<p_{2}
$$

and $s_{2}$ is the positive root of the quadratic equation

$$
s^{2}+5 s_{c}^{(2)} s+\left(s_{c}^{(2)}\right)^{2}-7 s_{c}^{(2)}=0 .
$$

Then the solution $u$ to $i u_{t}+\Delta u=|u|^{p_{1}} u+|u|^{p_{2}} u$ is global and scatters.

Acknowledgements The authors would like to thank Piotr Biler, Benjamin Dodson, Grzegorz Karch and the anonymous referee for comments and suggestions. This work is supported in part by the National Natural Science Foundation of China under grant No. 11171033, No. 11231006, and No. 11371059.

\section{REFERENCES}

[1] J. Bourgain, Scattering in the energy space and below for $3 D$ NLS. Journal d'Analyse Mathematique. 75 (1998), 267-297.

[2] J. Bourgain, Refinements of Strichartz' inequality and applications to $2 D-N L S$ with critical nonlinearity. Internat. Math. Res. Notices, 5 (1998), 253-283.

[3] J. Bourgain, Global well-posedness of defocusing $3 D$ critical NLS in the radial case. J. Amer. Math. Soc., 12 (1999), 145-171. MR1626257

[4] T. Cazenave, Semilinear Schrödinger equations. Courant Lecture Notes in Mathematics, Vol. 10. New York: New York University Courant Institute of Mathematical Sciences, 2003. ISBN: 0-8218-3399-5.

[5] T. Cazenave and F. Weissler, The Cauchy problem for the critical nonlinear Schrödinger equation in $H^{s}$. Nonlinear Anal., 14 (1990), 807-836.

[6] M. Christ and M. Weinstein, Dispersion of small amplitude solutions of the generalized Korteweg-de Vries equation. J. Funct. Anal., 100 (1991), 87-109.

[7] J. Colliander, M. Grillakis and N. Tzirakis, Improved interaction Morawetz inequalities for the cubic nonlinear Schrödinger equation on $\mathbb{R}^{2}$. Int. Math. Res. Not., 23 (2007), 90-119.

[8] J. Colliander, M. Grillakis and N. Tzirakis, Tensor products and correlation estimates with applications to nonlinear Schrödinger equations. Comm. Pure and Applied Math., 62 (2009), 920-968.

[9] J. Colliander, J. Holmer, M. Visan and X. Zhang, Global existence and scattering for rough solutions to generalized nonlinear Schrödinger equations on $\mathbb{R}$. Comm. Pure Appl. Anal., 7 (2008), 467-489.

[10] J. Colliander, M. Keel, G. Staffilani, H. Takaoka and T. Tao, Global well-posedness for Schrödinger equations with deriatives. SIAM J. Math. Anal., 33(2001), 649-669.

[11] J. Colliander, M. Keel, G. Staffilani, H. Takaoka and T. Tao, Almost conservation laws and global rough solutions to a nonlinear Schrödinger equation. Math. Res. Lett., 9 (2002), 659-682. 
[12] J. Colliander, M. Keel, G. Staffilani, H. Takaoka and T. Tao, A refined global well-posedness result for the Schrödinger equations with derivative. SIAM J. Math. Anal., 34 (2002), 64-86.

[13] J. Colliander, M. Keel, G. Staffilani, H. Takaoka and T. Tao, Polynomial upper bounds for the orbit instability of the $1 D$ cubic NLS below the energy norm. Discrete Contin. Dyn. Syst., 9 (2003), 31-54.

[14] J. Colliander, M. Keel, G. Staffilani, H. Takaoka and T. Tao, Polynomial upper bounds for the instability of the nonlinear Schrödinger equation below the energy norm. Commun. Pure Appl. Anal., 2 (2003), 33-50.

[15] J. Colliander, M. Keel, G. Staffilani, H. Takaoka and T. Tao, Resonant decompositions and the I-method for cubic nonlinear Schrödinger equation on $\mathbb{R}^{2}$. Discrete and Continuous Dynamical Systems A, 21 (2007), 665-696.

[16] J. Colliander, M. Keel, G. Staffilani, H. Takaoka and T. Tao, Global existence and scattering for rough solutions to a nonlinear Schrödinger equation on $\mathbb{R}^{3}$, Comm. Pure Appl. Math., 57 (2004), 987-1014.

[17] J. Colliander, M. Keel, G. Staffilani, H. Takaoka, and T. Tao, Global well-posedness and scattering for the energy-critical nonlinear Schrödinger equation in $\mathbb{R}^{3}$. Ann. Math., 167 (2008), 767-865.

[18] J. Colliander and T. Roy, Bootstrapped Morawetz estimates and resonant decomposition for low regularity global solutions of cubic $N L S$ on $\mathbb{R}^{2}$. Comm. Pure Appl. Anal., 10 (2011), 397-414.

[19] B. Dodson, Improved almost Morawetz estimates for the cubic nonlinear Schrödinger equation. Comm. Pure Appl. Anal., 10 (2011), 127-140.

[20] B. Dodson, Global well-posedness and scattering for the defocusing, cubic nonlinear Schrödinger equation when $n=3$ via a linear-nonlinear decomposition, Discrete and Continuous Dynamical Systems. Series A, 33 (2013), 1905-1926.

[21] B. Dodson, Global well-posedness and scattering for nonlinear Schrödinger equations with algebraic nonlinearity when $d=2,3, u_{0}$ radial, arXiv: $1405.0218 \mathrm{v} 1$.

[22] B. Dodson, Global well-posedness and scattering for the defocusing, L2-critical, nonlinear Schrödinger equation when $d \geqslant 3$. J. Amer. Math. Soc. 25 (2012), 429-463.

[23] B. Dodson, Global well-posedness and scattering for the defocusing, $L^{2}$-critical, nonlinear Schrödinger equation when $d=2$. Preprint arXiv:1006.1375.

[24] B. Dodson, Global well-posedness and scattering for the defocusing, $L^{2}$-critical, nonlinear Schrödinger equation when $d=1$. Preprint arXiv: 1010.0040 .

[25] J. Ginibre and G. Velo, Scattering theory in the energy space for a class of nonlinear Schrödinger equations, J. Math. Pure Appl., 64 (1985), 363-401.

[26] J. Ginibre and G. Velo, Smoothing properties and retarded estimates for some dispersive evolution equations. Comm. Math. Phys., 144 (1992), 163-188.

[27] J. Ginibre and G. Velo, Quadratic Morawetz inequalities and asymptotic completeness in the energy space for nonlinear Schrödinger and Hartree equations, Quart. Appl. Math., 68 (2010), 113-134.

[28] M. Keel and T. Tao, Endpoint Strichartz estimates. Amer. J. Math., 120 (1998), 955-980.

[29] M. Keel and T. Tao, Local and global well posedness of wave maps on $\mathbb{R}^{1+1}$ for rough data, Int. Math. Res. Not., 21 (1998), 1117-1156.

[30] H. Koch and D. Tataru, A priori bounds for the $1 D$ cubic NLS in negative Sobolev spaces, Int. Math. Res. Not., 16 (2007), Art. ID rnm053,36pp.

[31] H. Koch and D. Tataru, Energy and local energy bounds for the $1 D$ cubic NLS equation in $H^{-\frac{1}{4}}$, Ann. de l'Institut Henri Poincaré. Analyse Non Linéaire, 29 (2012), 955-988.

[32] J. Lin and W. Strauss, Decay and scattering of solutions of a nonlinear Schrödinger equation, J. Func. Anal., 30 (1978), 245-263.

[33] C. Morawetz, Time decay for the nonlinear Klein-Gordon equation, Proc. Roy. Soc. A206 (1968), $291-296$.

[34] C. Morawetz and W. Strauss, Decay and scattering of solutions of a nonlinear relativistic wave equation, Comm. Pure Appl. Math., 25 (1972), 1-31.

[35] K. Nakanishi, Energy scattering for nonlinear Klein-Gordon and Schrödinger equations in spatial dimensions 1 and 2, J. Funct. Anal., 169 (1999), 201-225.

[36] F. Planchon and L. Vega, Bilinear virial identities and applications, Ann. Sci. Ecole Normale Supérieure, Quatrième Série, 42 (2009), 261-290.

[37] R. S. Strichartz, Restriction of Fourier transform to quadratic surfaces and decay of solutions of wave equations. Duke Math. J., 44 (1977), 705-774.

[38] Q. Su, Global well-posedness and scattering for the defocusing, cubic $N L S$ in $\mathbb{R}^{3}$, Math. Res. Lett., 19 (2012), 431-451.

[39] T. Tao, M. Visan and X. Zhang, The nonlinear Schrödinger equation with combined power-type nonlinearities. Comm. Part. Diff. Eq., 32 (2007), 1281-1343.

[40] N. Tzirakis, The Cauchy problem for the semilinear quintic Schrödinger equation in one dimension, the defocusing case, Diff. Int. Eq., 18 (2005), 947-960. 
[41] M. Visan and X. Zhang, Global well-posedness and scattering for a class of nonlinear Schrödinger equations below the energy space. Diff. Int. Eq., 22 (2009), 99-124.

Institute of Applied Physics and Computational Mathematics, P.O. Box 8009, Beijing 100088, P.R. China., and Beijing Center for Mathematics and Information Interdisciplinary Sciences, Beijing, 100048, P.R.CHINA.

E-mail address: miao_changxing@iapcm.ac.cn

The Graduate School of China Academy of Engineering Physics, P. O. Box 2101, Beijing, China, 100088

E-mail address: zhengjiqiang@gmail.com 